\title{
Non-autonomous random oscillating systems of the fourth order under small periodical external perturbations with jumps
}

\author{
Olga Borysenko ${ }^{1}$, Oleksandr Borysenko ${ }^{2, *}$ \\ ${ }^{1}$ Department of Mathematical Physics, National Technical University of Ukraine, Ukraine \\ ${ }^{2}$ Department of Probability Theory, Statistics and Actuarial Mathematics, Taras Shevchenko National University of Kyiv, Ukraine
}

\begin{abstract}
The asymptotic behavior of a non-autonomous oscillating system described by a differential equation of the fourth order with small non-linear periodical external perturbations of "white noise", non-centered and centered "Poisson noise" types is studied. Each term of external perturbations has own order of a small parameter $\varepsilon$. If the small parameter is equal to zero, then the general solution of the obtained non-stochastic fourth order differential equation has an oscillating part. We consider the given differential equation with external stochastic perturbations as the system of stochastic differential equations and study the limit behavior of its solution at the time moment $t / \varepsilon^{k}$, as $\varepsilon \rightarrow 0$. The system of averaging stochastic differential equations is derived and its dependence on the order of the small parameter in each term of external perturbations is studied. The non-resonance and resonance cases are considered.
\end{abstract}

Keywords Asymptotic Behavior, Non-Autonomous Oscillating System, Stochastic Differential Equation, Non-Resonance Case, Resonance, Periodical Disturbances

AMS 2010 subject classifications. Primary: 34C15, 60H10 Secondary: 34F05, 74Q10

DOI: $10.19139 /$ soic.v7i1.459

\section{Introduction}

Studying of oscillation processes has a great importance in different areas of mechanics, physics, technics, and economics. As examples of the oscillation systems we can consider vibration of constructions and mechanisms, electromagnetic oscillations in radio-technology and optics, auto-oscillation in control systems, sound and ultrasound vibrations. It worth to mention that oscillatory models in finance are studied in Ping Chen, Sardar M.N. Islam [1] and in C. Ye, J.P. Huang [2].

The averaging method proposed by N.M.Krylov, N.N.Bogolyubov and Yu.A.Mytropolskij ([3], [4]) is one of the main tool in studying of the deterministic oscillating systems under the action of a small non-linear perturbations. The case of small random "white noise" type disturbances in oscillating systems of the second order is considered in the paper of Yu.A.Mytropolskij, V.G.Kolomiets [5]. The autonomous and non-autonomous oscillating systems of the second order under the action of "white noise" and Poisson type noise perturbations are studied in the papers of O.V.Borysenko ([6], [7]). The particular case of the third order oscillating systems are investigated in the articles of O.D.Borysenko, O.V.Borysenko [8], O.D.Borysenko, O.V.Borysenko and I.G.Malyshev ([9], [10]). The limit behavior of autonomous and non-autonomous third order oscillating system under the action of an external small nonlinear random disturbances such as multidimensional "white noise" and "Poisson noise" was studied in ([11], [12]). The autonomous forth order stochastic oscillating systems is considered in the papers of

\footnotetext{
${ }^{*}$ Correspondence to: Oleksandr Borysenko (Email: odb@univ.kiev.ua). Department of Probability Theory, Statistics and Actuarial
} Mathematics, Taras Shevchenko National University of Kyiv, Volodymyrska 64 Str., Kyiv 01601, Ukraine.

ISSN 2310-5070 (online) ISSN 2311-004X (print)

Copyright (C) 2019 International Academic Press 
O.D.Borysenko, O.V.Borysenko ([13] - [15]). The averaging method on the infinite interval for the system of Ito stochastic differential equations is studied in [16].

The non-autonomous oscillating systems of the fours order under the action of "white noise", centered and noncentered Poisson type noises perturbations are studied in ([17], [18]). It is considered the behavior, as $\varepsilon \rightarrow 0$, of the oscillating system driven by stochastic differential equation

$$
\begin{aligned}
& x^{I V}(t)+b_{1} x^{\prime \prime \prime}(t)+b_{2} x^{\prime \prime}(t)+b_{3} x^{\prime}(t)+b_{4} x(t)= \\
& =\varepsilon^{k_{0}} f_{0}\left(\mu_{0} t, x(t), x^{\prime}(t), x^{\prime \prime}(t), x^{\prime \prime \prime}(s)\right)+f_{\varepsilon}\left(t, x(t), x^{\prime}(t), x^{\prime \prime}(t), x^{\prime \prime \prime}(s)\right)
\end{aligned}
$$

with non-random initial conditions $x(0)=x_{0}^{(1)}, x^{\prime}(0)=x_{0}^{(2)}, x^{\prime \prime}(0)=x_{0}^{(3)}, x^{\prime \prime \prime}(0)=x_{0}^{(4)}$, where $\varepsilon>0$ is a small parameter, $f_{\varepsilon}(t, \mathbf{x}(t)), \mathbf{x}(t)=\left(x(t), x^{\prime}(t), x^{\prime \prime}(t), x^{\prime \prime \prime}(t)\right)$ is a random function such that

$$
\begin{aligned}
\int_{0}^{t} f_{\varepsilon}(s, \mathbf{x}(s)) d s & =\sum_{i=1}^{m} \varepsilon^{k_{i}} \int_{0}^{t} f_{i}\left(\mu_{i} s, \mathbf{x}(s)\right) d w_{i}(s)+\varepsilon^{k_{m+1}} \int_{0}^{t} \int_{\mathbb{R}} f_{m+1}\left(\mu_{m+1} s, \mathbf{x}(s), z\right) \tilde{\nu}_{1}(d s, d z) \\
& +\varepsilon^{k_{m+2}} \int_{0}^{t} \int_{\mathbb{R}} f_{m+2}\left(\mu_{m+2} s, \mathbf{x}(s), z\right) \nu_{2}(d s, d z),
\end{aligned}
$$

$k_{i}>0, i=\overline{0, m+2} ; \quad f_{i}, i=\overline{0, m+2}$ are non-random functions periodic on $\mu_{i} t, i=\overline{0, m+2}$ with period $2 \pi ; w_{i}(t), i=\overline{1, m}$ are independent one-dimensional Wiener processes; $\tilde{\nu}_{i}(d t, d y)=\nu_{i}(d t, d y)-\Pi_{i}(d y) d t$, $E \nu_{i}(d t, d y)=\Pi_{i}(d y) d t, i=1,2 ; \nu_{i}(d t, d y), i=1,2$ are the independent Poisson measures independent on $w_{i}(t), i=\overline{1, m} ; \Pi_{i}(A), i=1,2$ are a finite measures on Borel sets in $\mathbb{R}$.

We will study the asymptotic behavior of the oscillating system (1), as $\varepsilon \rightarrow 0$, in the case when there exists stable harmonic oscillations at the system under condition $\varepsilon=0$. Under this condition corresponding characteristic equation has a form

$$
\lambda^{4}+b_{1} \lambda^{3}+b_{2} \lambda^{2}+b_{3} \lambda+b_{4}=0
$$

The following cases were considered previously:

1) ([17]) $b_{1}>0, b_{3}>0, b_{1} b_{2}>b_{3}, b_{1}^{2}>4\left(b_{2}-\frac{b_{3}}{b_{1}}\right), b_{1}^{2} b_{4}=b_{3}\left(b_{1} b_{2}-b_{3}\right)$. In this case the characteristic equation has a roots

$$
\begin{aligned}
& \lambda_{1}=-\eta_{1}, \lambda_{2}=-\eta_{2}, \lambda_{3,4}= \pm i \omega, \text { where } \\
& \eta_{1,2}=\frac{1}{2}\left(b_{1} \pm \sqrt{b_{1}^{2}-4\left(b_{2}-\frac{b_{3}}{b_{1}}\right)}\right), \omega^{2}=\frac{b_{3}}{b_{1}} .
\end{aligned}
$$

2) ([18]) $b_{1}>0, b_{3}>0, b_{4}>b_{1} b_{3} / 4, b_{1}^{2} b_{4}=b_{3}\left(b_{1} b_{2}-b_{3}\right)$. In this case the characteristic equation has a roots

$$
\begin{aligned}
& \lambda_{1}=-\eta+i \nu, \lambda_{2}=-\eta-i \nu, \lambda_{3,4}= \pm i \omega, \text { where } \\
& \eta=\frac{b_{1}}{2}, \nu=\frac{1}{2} \sqrt{\frac{b_{1}\left(4 b_{4}-b_{1} b_{3}\right)}{b_{3}}}, \omega^{2}=\frac{b_{3}}{b_{1}} .
\end{aligned}
$$

The main results of this paper are following. We investigate the asymptotic behavior of the oscillating system (1), as $\varepsilon \rightarrow 0$, in the case when the characteristic equation has multiple real root and two conjugate pure imaginary roots (Theorem 3), and in the case of two pairs of imaginary adjoined roots of the characteristic equation (Theorem 4). In both situations, we consider the non-resonance and resonance cases. 
We will consider the equation (1) as the system of stochastic differential equations

$$
\begin{aligned}
& d y_{i}(t)=y_{i+1}(t) d t, i=\overline{1,3} \\
& d y_{4}(t)=\left[-(\mathbf{b} \cdot \mathbf{y}(t))+\varepsilon^{k_{0}} f_{0}\left(\mu_{0} t, \mathbf{y}(t)\right)+\varepsilon^{k_{m+2}} \int_{\mathbb{R}} f_{m+2}\left(\mu_{m+2} t, \mathbf{y}(t), z\right) \Pi_{2}(d z)\right] d t \\
& +\sum_{i=1}^{m} \varepsilon^{k_{i}} f_{i}\left(\mu_{i} t, \mathbf{y}(t)\right) d w_{i}(t)+\varepsilon^{k_{m+1}} \int_{\mathbb{R}} f_{m+1}\left(\mu_{m+1} t, \mathbf{y}(t), z\right) \tilde{\nu}_{1}(d t, d z) \\
& +\varepsilon^{k_{m+2}} \int_{\mathbb{R}} f_{m+2}\left(\mu_{m+2} t, \mathbf{y}(t), z\right) \tilde{\nu}_{2}(d t, d z),
\end{aligned}
$$

$\mathbf{y}(t)=\left(y_{1}(t), \ldots, y_{4}(t)\right), \mathbf{b}=\left(b_{4}, b_{3}, b_{2}, b_{1}\right), y_{i}(0)=x_{0}^{(i)}, i=\overline{1,4},(\mathbf{b} \cdot \mathbf{y}(t))-$ is an inner product of vectors $\mathbf{b}$ and $\mathbf{y}(t)$.

The rest of this paper is organized as follows. In Section 2, we present the previously obtained results deals with cases 1) and 2) for equation (1). In Section 3, we will study the case of multiple real root and two conjugate pure imaginary roots of the characteristic equation, and in Section 4, we consider the case of two pairs of imaginary adjoined roots of the characteristic equation.

In what follows we will use the constant $K>0$ for the notation of different constants, which do not depend on $\varepsilon$.

\section{Previously obtained results}

Case 1) ([17]). If $\varepsilon=0$, then the equation (1) has general solution in the form

$$
x(t)=C_{1} e^{-\eta_{1} t}+C_{2} e^{-\eta_{2} t}+A_{1} \cos \omega t+A_{2} \sin \omega t
$$

Let us denote

$$
C(t)=\left(C_{1}(t), C_{2}(t), A_{1}(t), A_{2}(t)\right), \quad \Theta(t)=\left(e^{-\eta_{1} t}, e^{-\eta_{2} t}, \cos \omega t, \sin \omega t\right),
$$

and let us consider the following representation of the solution $\mathbf{y}(t)$ to the system (2):

$$
y_{i}(t)=\left(C(t) \cdot \frac{d^{i-1}}{d t^{i-1}} \Theta(t)\right), i=\overline{1,4} .
$$

We can solve the system of linear equations (3) with respect to $\left(N_{1}(t), N_{2}(t), A_{1}(t), A_{2}(t)\right)$, where $N_{i}(t)=$ $C_{i}(t) e^{-\eta_{i} t}, i=1,2$ and using the Ito formula we derive the system of stochastic differential equations:

$$
\begin{aligned}
& d N_{1}(t)=-\eta_{1} N_{1}(t) d t+\frac{1}{\left(\eta_{2}-\eta_{1}\right)\left(\eta_{1}^{2}+\omega^{2}\right)} d H(t), \\
& d N_{2}(t)=-\eta_{2} N_{2}(t) d t-\frac{1}{\left(\eta_{2}-\eta_{1}\right)\left(\eta_{2}^{2}+\omega^{2}\right)} d H(t), \\
& d A_{1}(t)=\frac{-\omega\left(\eta_{1}+\eta_{2}\right) \cos \omega t+\left(\omega^{2}-\eta_{1} \eta_{2}\right) \sin \omega t}{\omega\left(\eta_{1}^{2}+\omega^{2}\right)\left(\eta_{2}^{2}+\omega^{2}\right)} d H(t), \\
& d A_{2}(t)=\frac{-\omega\left(\eta_{1}+\eta_{2}\right) \sin \omega t-\left(\omega^{2}-\eta_{1} \eta_{2}\right) \cos \omega t}{\omega\left(\eta_{1}^{2}+\omega^{2}\right)\left(\eta_{2}^{2}+\omega^{2}\right)} d H(t),
\end{aligned}
$$




$$
\begin{aligned}
& d H(t)=\left[\varepsilon^{k_{0}} \tilde{f}_{0}\left(\mu_{0} t, N(t), A(t), \omega t\right)+\varepsilon^{k_{m+2}} \int_{\mathbb{R}} \tilde{f}_{m+2}\left(\mu_{m+2} t, N(t), A(t), \omega t, z\right) \Pi_{2}(d z)\right] d t \\
& +\sum_{i=1}^{m} \varepsilon^{k_{i}} \tilde{f}_{i}\left(\mu_{i} t, N(t), A(t), \omega t\right) d w_{i}(t)+\varepsilon^{k_{m+1}} \int_{\mathbb{R}} \tilde{f}_{m+1}\left(\mu_{m+1} t, N(t), A(t), \omega t, z\right) \tilde{\nu}_{1}(d t, d z) \\
& +\varepsilon^{k_{m+2}} \int_{\mathbb{R}} \tilde{f}_{m+2}\left(\mu_{m+2} t, N(t), A(t), \omega t, z\right) \tilde{\nu}_{2}(d t, d z),
\end{aligned}
$$

where $N(t)=\left(N_{1}(t), N_{2}(t)\right), \quad A(t)=\left(A_{1}(t), A_{2}(t)\right) ; \quad \tilde{f}_{i}\left(\mu_{i} t, N(t), A(t), \omega t\right), \quad i=\overline{0, m}$ are obtained from $f_{i}\left(\mu_{i} t, \mathbf{y}(t)\right), i=\overline{0, m}$ and $\tilde{f}_{i}\left(\mu_{i} t, N(t), A(t), \omega t, z\right), i=m+1, m+2$ are obtained from $f_{i}\left(\mu_{i} t, \mathbf{y}(t), z\right), i=$ $m+1, m+2$ using (3).

\section{Theorem 1}

([17]) Let $\Pi_{i}(\mathbb{R})<\infty, i=1,2, t \in\left[0, t_{0}\right], k=\min \left(k_{0}, 2 k_{1}, \ldots, 2 k_{m+1}, k_{m+2}\right)$. Let us suppose, that functions $f_{j}, j=\overline{0, m+2}$ bounded and satisfy Lipschitz condition on $y_{i}, i=\overline{1,4}$. If given below matrix $\bar{\sigma}^{2}\left(A_{1}, A_{2}\right)$ is nonnegative definite, then:

1. Let $\mu_{i}=\frac{p_{i}}{q_{i}} \omega$ for all $i=\overline{0, m+2}$, where $p_{i}$ and $q_{i}$ are some relatively prime integers. If $k_{0}=2 k_{i}=k_{m+2}, i=$ $\overline{1, m+1}$, then the stochastic process $\xi_{\varepsilon}(t)=\left(N_{1}\left(t / \varepsilon^{k}\right), N_{2}\left(t / \varepsilon^{k}\right), A_{1}\left(t / \varepsilon^{k}\right), A_{2}\left(t / \varepsilon^{k}\right)\right)$ weakly converges, as $\varepsilon \rightarrow 0$, to the stochastic process $\bar{\xi}(t)=\left(0,0, \bar{A}_{1}(t), \bar{A}_{2}(t)\right)$, where $\bar{A}(t)=\left(\bar{A}_{1}(t), \bar{A}_{2}(t)\right)$ is the solution to the system of stochastic differential equations

$$
d \bar{A}(t)=\bar{\alpha}(\bar{A}(t)) d t+\bar{\sigma}(\bar{A}(t)) d \bar{w}(t), \quad \bar{A}(0)=\left(A_{1}(0), A_{2}(0)\right),
$$

where

$$
\begin{gathered}
\bar{\alpha}\left(A_{1}, A_{2}\right)=\frac{1}{4 \pi^{2}} \sum_{p_{0} n+q_{0} l=0} \int_{0}^{2 \pi} \int_{0}^{2 \pi} \hat{f}_{0}\left(\psi, A_{1}, A_{2}, \phi\right) \Psi(\phi) e^{-i(n \psi+l \phi)} d \phi d \psi \\
\left.+\sum_{p_{m+2} n+q_{m+2} l=0} \int_{0}^{2 \pi} \int_{0}^{2 \pi} \int_{\mathbb{R}} \hat{f}_{m+2}\left(\psi, A_{1}, A_{2}, \phi, z\right) \Psi(\phi) e^{-i(n \psi+l \phi)} \Pi_{2}(d z) d \phi d \psi\right], \\
\bar{\sigma}^{2}\left(A_{1}, A_{2}\right)=\frac{1}{4 \pi^{2}}\left[\sum_{j=1}^{m} \sum_{p_{j} n+q_{j} l=0} \int_{0}^{2 \pi} \int_{0}^{2 \pi} \hat{f}_{j}^{2}\left(\psi, A_{1}, A_{2}, \phi\right) \Psi(\phi) \Psi^{T}(\phi) e^{-i(n \psi+l \phi)} d \phi d \psi\right. \\
\left.+\sum_{p_{m+1} n+q_{m+1} l=0} \int_{0}^{2 \pi} \int_{0}^{2 \pi} \int_{\mathbb{R}} \hat{f}_{m+1}^{2}\left(\psi, A_{1}, A_{2}, \phi, z\right) \Psi(\phi) \Psi^{T}(\phi) e^{-i(n \psi+l \phi)} \Pi_{1}(d z) d \phi d \psi\right] \\
\Psi(\phi)=\frac{\omega^{2}}{\omega\left(\eta_{1}^{2}+\omega^{2}\right)\left(\eta_{2}^{2}+\omega^{2}\right)}\left(\begin{array}{l}
-\omega\left(\eta_{1}+\eta_{2}\right) \cos \phi+\left(\omega^{2}-\eta_{1} \eta_{2}\right) \sin \phi \\
-\omega\left(\eta_{1}\right) \sin \phi-\left(\omega^{2}-\eta_{1} \eta_{2}\right) \cos \phi
\end{array}\right), \\
\hat{f}_{j}\left(\psi, A_{1}, A_{2}, \phi\right)=\tilde{f}_{j}\left(\psi, 0,0, A_{1}, A_{2}, \phi\right), j=\overline{0, m},
\end{gathered}
$$

$\Psi^{T}(\phi)$ is the vector transpose to the vector $\Psi(\phi), \bar{w}(t)=\left(\bar{w}_{i}(t), i=1,2\right), \bar{w}_{i}(t), i=1,2$ are independent onedimensional Wiener processes.

2. If $k<k_{0}$ then in the averaging equation (4) we must put $\tilde{f}_{0} \equiv 0$; if $k<2 k_{i}$ for some $i=\overline{1, m}$, then in the averaging equation (4) we must put $\tilde{f}_{i} \equiv 0$ for such $i$; if $k<2 k_{m+1}$ then in the averaging equation (4) we must put $\tilde{f}_{m+1} \equiv 0$; if $k<k_{m+2}$ then in the averaging equation (4) we must put $\tilde{f}_{m+2} \equiv 0$.

3. If $\mu_{j} \neq \frac{p_{j}}{q_{j}} \omega$ for some $j=\overline{0, m+2}$ and any relatively prime integers $p_{j}$ and $q_{j}$, then in averaging coefficients in (4) we must put $l=n=0$ in corresponding sums containing $\hat{f}_{j}$. 
Case 2) ([18]). If $\varepsilon=0$, then the equation (1) has general solution in the form

$$
x(t)=e^{-\eta t}\left(C_{1} \cos \nu t+C_{2} \sin \nu t\right)+A_{1} \cos \omega t+A_{2} \sin \omega t .
$$

Let us denote

$$
C(t)=\left(C_{1}(t), C_{2}(t), A_{1}(t), A_{2}(t)\right), \quad \Phi(t)=\left(e^{-\eta t} \cos \nu t, e^{-\eta t} \sin \nu t, \cos \omega t, \sin \omega t\right),
$$

and let us consider the following representation of the solution $\mathbf{y}(t)$ to the system $(2)$ :

$$
y_{i}(t)=\left(C(t) \cdot \frac{d^{i-1}}{d t^{i-1}} \Phi(t)\right), i=\overline{1,4} .
$$

We can solve the system of linear equations (5) with respect to $\left(N_{1}(t), N_{2}(t), A_{1}(t), A_{2}(t)\right)$, where $N_{i}(t)=$ $C_{i}(t) e^{-\eta t}, i=1,2$ and using the Ito formula we derive the system of stochastic differential equations:

$$
\begin{aligned}
& d N_{1}(t)=-\eta N_{1}(t) d t+\frac{2 \eta \nu \cos \nu t+\left(\nu^{2}-\omega^{2}-\eta^{2}\right) \sin \nu t}{\nu\left[\left(\eta^{2}+\nu^{2}\right)^{2}+2\left(\eta^{2}-\nu^{2}\right) \omega^{2}+\omega^{4}\right]} d H(t), \\
& d N_{2}(t)=-\eta N_{2}(t) d t+\frac{2 \eta \nu \sin \nu t-\left(\nu^{2}-\omega^{2}-\eta^{2}\right) \cos \nu t}{\nu\left[\left(\eta^{2}+\nu^{2}\right)^{2}+2\left(\eta^{2}-\nu^{2}\right) \omega^{2}+\omega^{4}\right]} d H(t), \\
& d A_{1}(t)=-\frac{2 \eta \omega \cos \omega t+\left(\eta^{2}+\nu^{2}-\omega^{2}\right) \sin \omega t}{\omega\left[\left(\eta^{2}+\nu^{2}\right)^{2}+2\left(\eta^{2}-\nu^{2}\right) \omega^{2}+\omega^{4}\right]} d H(t), \\
& d A_{2}(t)=\frac{-2 \eta \omega \sin \omega t+\left(\eta^{2}+\nu^{2}-\omega^{2}\right) \cos \omega t}{\omega\left[\left(\eta^{2}+\nu^{2}\right)^{2}+2\left(\eta^{2}-\nu^{2}\right) \omega^{2}+\omega^{4}\right]} d H(t), \\
& d H(t)=\left[\varepsilon^{k_{0}} \tilde{f}_{0}\left(\mu_{0} t, N(t), A(t), \omega t\right)+\varepsilon^{k_{m+2}} \int_{\mathbb{R}} \tilde{f}_{m+2}\left(\mu_{m+2} t, N(t), A(t), \omega t, z\right) \Pi_{2}(d z)\right] d t \\
& +\sum_{i=1}^{m} \varepsilon^{k_{i}} \tilde{f}_{i}\left(\mu_{i} t, N(t), A(t), \omega t\right) d w_{i}(t)+\varepsilon^{k_{m+1}} \int_{\mathbb{R}} \tilde{f}_{m+1}\left(\mu_{m+1} t, N(t), A(t), \omega t, z\right) \tilde{\nu}_{1}(d t, d z) \\
& +\varepsilon^{k_{m+2}} \int_{\mathbb{R}} \tilde{f}_{m+2}\left(\mu_{m+2} t, N(t), A(t), \omega t, z\right) \tilde{\nu}_{2}(d t, d z),
\end{aligned}
$$

where $N(t)=\left(N_{1}(t), N_{2}(t)\right), \quad A(t)=\left(A_{1}(t), A_{2}(t)\right) ; \quad \tilde{f}_{i}\left(\mu_{i} t, N(t), A(t), \omega t\right), \quad i=\overline{0, m}$ are obtained from $f_{i}\left(\mu_{i} t, \mathbf{y}(t)\right), i=\overline{0, m}$ and $\tilde{f}_{i}\left(\mu_{i} t, N(t), A(t), \omega t, z\right), i=m+1, m+2$ are obtained from $f_{i}\left(\mu_{i} t, \mathbf{y}(t), z\right), i=$ $m+1, m+2$ using (5).

\section{Theorem 2}

([18]) Let $\Pi_{i}(\mathbb{R})<\infty, i=1,2, t \in\left[0, t_{0}\right], k=\min \left(k_{0}, 2 k_{1}, \ldots, 2 k_{m+1}, k_{m+2}\right)$. Let us suppose, that functions $f_{j}, j=\overline{0, m+2}$ bounded and satisfy Lipschitz condition on $y_{i}, i=\overline{1,4}$. If given below matrix $\bar{\sigma}^{2}\left(A_{1}, A_{2}\right)$ is nonnegative definite, then:

1. Let $\mu_{i}=\frac{p_{i}}{q_{i}} \omega$ for all $i=\overline{0, m+2}$, where $p_{i}$ and $q_{i}$ are some relatively prime integers. If $k_{0}=2 k_{i}=k_{m+2}, i=$ $\overline{1, m+1}$, then the stochastic process $\xi_{\varepsilon}(t)=\left(N_{1}\left(t / \varepsilon^{k}\right), N_{2}\left(t / \varepsilon^{k}\right), A_{1}\left(t / \varepsilon^{k}\right), A_{2}\left(t / \varepsilon^{k}\right)\right)$ weakly converges, as $\varepsilon \rightarrow 0$, to the stochastic process $\bar{\xi}(t)=\left(0,0, \bar{A}_{1}(t), \bar{A}_{2}(t)\right)$, where $\bar{A}(t)=\left(\bar{A}_{1}(t), \bar{A}_{2}(t)\right)$ is the solution to the system of stochastic differential equations

$$
d \bar{A}(t)=\bar{\alpha}(\bar{A}(t)) d t+\bar{\sigma}(\bar{A}(t)) d \bar{w}(t), \quad \bar{A}(0)=\left(A_{1}(0), A_{2}(0)\right),
$$

where

$$
\begin{aligned}
& \bar{\alpha}\left(A_{1}, A_{2}\right)=\frac{1}{4 \pi^{2}}\left[\sum_{p_{0} n+q_{0} l=0} \int_{0}^{2 \pi} \int_{0}^{2 \pi} \hat{f}_{0}\left(\psi, A_{1}, A_{2}, \phi\right) \Upsilon(\phi) e^{-i(n \psi+l \phi)} d \phi d \psi\right. \\
& \left.+\sum_{p_{m+2} n+q_{m+2} l=0} \int_{0}^{2 \pi} \int_{0}^{2 \pi} \int_{\mathbb{R}} \hat{f}_{m+2}\left(\psi, A_{1}, A_{2}, \phi, z\right) \Upsilon(\phi) e^{-i(n \psi+l \phi)} \Pi_{2}(d z) d \phi d \psi\right],
\end{aligned}
$$




$$
\begin{gathered}
\bar{\sigma}^{2}\left(A_{1}, A_{2}\right)=\frac{1}{4 \pi^{2}}\left[\sum_{j=1}^{m} \sum_{p_{j} n+q_{j} l=0} \int_{0}^{2 \pi} \int_{0}^{2 \pi} \hat{f}_{j}^{2}\left(\psi, A_{1}, A_{2}, \phi\right) \Upsilon(\phi) \Upsilon^{T}(\phi) e^{-i(n \psi+l \phi)} d \phi d \psi+\right. \\
\left.+\sum_{p_{m+1}} \int_{0+q_{m+1} l=0}^{2 \pi} \int_{0}^{2 \pi} \int_{\mathbb{R}} \hat{f}_{m+1}^{2}\left(\psi, A_{1}, A_{2}, \phi, z\right) \Upsilon(\phi) \Upsilon^{T}(\phi) e^{-i(n \psi+l \phi)} \Pi_{1}(d z) d \phi d \psi\right], \\
\Upsilon(\phi)=\frac{1}{\omega\left[\left(\eta^{2}+\nu^{2}\right)^{2}+2\left(\eta^{2}-\nu^{2}\right) \omega^{2}+\omega^{4}\right]}\left(\begin{array}{c}
-2 \eta \omega \cos \phi-\left(\eta^{2}+\nu^{2}-\omega^{2}\right) \sin \phi \\
-2 \eta \omega \sin \phi+\left(\eta^{2}+\nu^{2}-\omega^{2}\right) \cos \phi
\end{array}\right)
\end{gathered}
$$

$\Upsilon^{T}(\phi)$ is the vector transpose to the vector $\Upsilon(\phi), \bar{w}(t)=\left(\bar{w}_{i}(t), i=1,2\right), \bar{w}_{i}(t), i=1,2$ are independent onedimensional Wiener processes.

2. If $k<k_{0}$ then in the averaging equation (6) we must put $\tilde{f}_{0} \equiv 0$; if $k<2 k_{i}$ for some $i=\overline{1, m}$, then in the averaging equation (6) we must put $\tilde{f}_{i} \equiv 0$ for such $i$; if $k<2 k_{m+1}$ then in the averaging equation (6) we must put $\tilde{f}_{m+1} \equiv 0$; if $k<k_{m+2}$ then in the averaging equation (6) we must put $\tilde{f}_{m+2} \equiv 0$.

3. If $\mu_{j} \neq \frac{p_{j}}{q_{j}} \omega$ for some $j=\overline{0, m+2}$ and any relatively prime integers $p_{j}$ and $q_{j}$, then in averaging coefficients in (6) we must put $l=n=0$ in corresponding sums containing $\hat{f}_{j}$.

\section{The case of multiple real root and two conjugate pure imaginary roots of characteristic equation}

From Borysenko O. and Malyshev I. [19], using the obvious modifications we obtain following results.

Lemma 1

Let for each $x \in \mathbb{R}^{d}$ there exists

$$
\lim _{T \rightarrow \infty} \frac{1}{T} \int_{A}^{T+A} f(t, x) d t=\bar{f}(x)
$$

uniformly with respect to $A$, the function $\bar{f}(x)$ is bounded and continuous, the function $f(t, x)$ is bounded and continuous in $x$ uniformly with respect to $(t, x)$ in any region $t \in[0, \infty),|x| \leq K$, and stochastic process $\xi(t) \in \mathbb{R}^{d}$ is continuous, then

$$
\lim _{\varepsilon \rightarrow 0} \int_{0}^{t} f\left(\frac{s}{\varepsilon}, \xi(s)\right) d s=\int_{0}^{t} \bar{f}(\xi(s)) d s
$$

almost surely for all arbitrary $t \in\left[0, t_{0}\right]$.

\section{Remark 1}

Let $f(t, x, z)$ is bounded and uniformly continuous in $x$ with respect to $t \in[0, \infty)$ and $z \in \mathbb{R}$ in every compact set $|x| \leq K, x \in \mathbb{R}^{d}$. Let $\Pi(\cdot)$ be a finite measure on the $\sigma$-algebra of Borel sets in $\mathbb{R}$ and let

$$
\lim _{T \rightarrow \infty} \frac{1}{T} \int_{A}^{T+A} f(t, x, z) d t=\bar{f}(x, z),
$$

uniformly with respect to $A$ for each $x \in \mathbb{R}^{d}, z \in \mathbb{R}$, where $\bar{f}(x, z)$ is bounded, uniformly continuous in $x$ with respect to $z \in \mathbb{R}$ in every compact set $|x| \leq K$. Then for any continuous process $\xi(t) \in \mathbb{R}^{d}$ we have

$$
\lim _{\varepsilon \rightarrow 0} \int_{0}^{t} \int_{\mathbb{R}} f\left(\frac{s}{\varepsilon}, \xi(s), z\right) \Pi(d z) d s=\int_{0}^{t} \int_{\mathbb{R}} \bar{f}(\xi(s), z) \Pi(d z) d s
$$

almost surely for all arbitrary $t \in\left[0, t_{0}\right]$.

In this section we will study the following case:

$$
b_{1}>0, \quad 4 b_{2}>b_{1}^{2}, \quad b_{3}=b_{1}\left(b_{2}-\frac{b_{1}^{2}}{4}\right), \quad b_{4}=b_{1}^{2} \frac{\left(b_{2}-\frac{b_{1}^{2}}{4}\right)}{4} .
$$


Characteristic equation has a roots

$$
\lambda_{1,2}=-\eta, \quad \lambda_{3,4}= \pm i \omega, \quad \text { where } \quad \eta=b_{1} / 2, \quad \omega^{2}=4 b_{4} / b_{1}^{2} .
$$

If $\varepsilon=0$ then the equation (1) has general solution in the form

$$
x(t)=C_{1} e^{-\eta t}+C_{2} t e^{-\eta t}+A_{1} \cos \omega t+A_{2} \sin \omega t .
$$

Let us consider the following representation of the solution $\mathbf{y}(t)$ to the system (2):

$$
\begin{aligned}
& y_{1}(t)=N_{1}(t)+A_{1}(t) \cos \omega t+A_{2}(t) \sin \omega t, \\
& y_{2}(t)=-\eta N_{1}(t)+N_{2}(t)-A_{1}(t) \omega \sin \omega t+A_{2}(t) \omega \cos \omega t, \\
& y_{3}(t)=\eta^{2} N_{1}(t)-2 \eta N_{2}(t)-A_{1}(t) \omega^{2} \cos \omega t-A_{2}(t) \omega^{2} \sin \omega t, \\
& y_{4}(t)=-\eta^{3} N_{1}(t)+3 \eta^{2} N_{2}(t)+A_{1}(t) \omega^{3} \sin \omega t-A_{2}(t) \omega^{3} \cos \omega t,
\end{aligned}
$$

where

$$
N_{1}(t)=\left(C_{1}(t)+t C_{2}(t)\right) e^{-\eta t}, N_{2}(t)=C_{2}(t) e^{-\eta t} .
$$

We can solve the system of linear equations (7) with respect to $\left(N_{1}(t), N_{2}(t), A_{1}(t), A_{2}(t)\right)$ and using the Ito formula we derive the system of stochastic differential equations:

$$
\begin{gathered}
d N_{1}(t)=\left[-\eta N_{1}(t)+N_{2}(t)\right] d t+\frac{2 \eta}{\left(\eta^{2}+\omega^{2}\right)^{2}} d H(t), \\
d N_{2}(t)=-\eta N_{2}(t) d t+\frac{1}{\left(\eta^{2}+\omega^{2}\right)} d H(t), \\
d A_{1}(t)=\frac{\left(\omega^{2}-\eta^{2}\right) \sin \omega t-2 \eta \omega \cos \omega t}{\omega\left(\eta^{2}+\omega^{2}\right)^{2}} d H(t), \\
d A_{2}(t)=-\frac{\left(\omega^{2}-\eta^{2}\right) \cos \omega t+2 \eta \omega \sin \omega t}{\omega\left(\eta^{2}+\omega^{2}\right)^{2}} d H(t), \\
d H(t)=\left[\begin{array}{c}
\left.\varepsilon^{k_{0}} \tilde{f}_{0}\left(\mu_{0} t, N(t), A(t), \omega t\right)+\varepsilon^{k_{m+2}} \int_{\mathbb{R}} \tilde{f}_{m+2}\left(\mu_{m+2} t, N(t), A(t), \omega t, z\right) \Pi_{2}(d z)\right] d t \\
+\sum_{i=1}^{m} \varepsilon^{k_{i}} \tilde{f}_{i}\left(\mu_{i} t, N(t), A(t), \omega t\right) d w_{i}(t)+\varepsilon^{k_{m+1}} \int_{\mathbb{R}} \tilde{f}_{m+1}\left(\mu_{m+1} t, N(t), A(t), \omega t, z\right) \tilde{\nu}_{1}(d t, d z) \\
+\varepsilon^{k_{m+2}} \int_{\mathbb{R}} \tilde{f}_{m+2}\left(\mu_{m+2} t, N(t), A(t), \omega t, z\right) \tilde{\nu}_{2}(d t, d z),
\end{array}\right.
\end{gathered}
$$

where $N(t)=\left(N_{1}(t), N_{2}(t)\right), \quad A(t)=\left(A_{1}(t), A_{2}(t)\right) ; \quad \tilde{f}_{i}\left(\mu_{i} t, N(t), A(t), \omega t\right), \quad i=\overline{0, m}$ are obtained from $f_{i}\left(\mu_{i} t, \mathbf{y}(t)\right), i=\overline{0, m}$ and $\tilde{f}_{i}\left(\mu_{i} t, N(t), A(t), \omega t, z\right), i=m+1, m+2$ are obtained from $f_{i}\left(\mu_{i} t, \mathbf{y}(t), z\right), i=$ $m+1, m+2$ using (7).

\section{Theorem 3}

Let $\Pi_{i}(\mathbb{R})<\infty, i=1,2, t \in\left[0, t_{0}\right], k=\min \left(k_{0}, 2 k_{1}, \ldots, 2 k_{m+1}, k_{m+2}\right)$. Let us suppose, that functions $f_{j}, j=$ $\overline{0, m+2}$ bounded and satisfy Lipschitz condition on $y_{i}, i=\overline{1,4}$. If given below matrix $\bar{\sigma}^{2}\left(A_{1}, A_{2}\right)$ is non-negative definite, then:

1. Let $\mu_{i}=\frac{p_{i}}{q_{i}} \omega$ for all $i=\overline{0, m+2}$, where $p_{i}$ and $q_{i}$ are some relatively prime integers. If $k_{0}=2 k_{i}=k_{m+2}, i=$ $\overline{1, m+1}$, then the stochastic process $\xi_{\varepsilon}(t)=\left(N_{1}\left(t / \varepsilon^{k}\right), N_{2}\left(t / \varepsilon^{k}\right), A_{1}\left(t / \varepsilon^{k}\right), A_{2}\left(t / \varepsilon^{k}\right)\right)$ weakly converges, as $\varepsilon \rightarrow 0$, to the stochastic process $\bar{\xi}(t)=\left(0,0, \bar{A}_{1}(t), \bar{A}_{2}(t)\right)$, where $\bar{A}(t)=\left(\bar{A}_{1}(t), \bar{A}_{2}(t)\right)$ is the solution to the system of stochastic differential equations

$$
d \bar{A}(t)=\bar{\alpha}(\bar{A}(t)) d t+\bar{\sigma}(\bar{A}(t)) d \bar{w}(t), \quad \bar{A}(0)=\left(A_{1}(0), A_{2}(0)\right),
$$


where

$$
\begin{gathered}
\bar{\alpha}\left(A_{1}, A_{2}\right)=\frac{1}{4 \pi^{2}} \sum_{p_{0} n+q_{0} l=0} \int_{0}^{2 \pi} \int_{0}^{2 \pi} \hat{f}_{0}\left(\psi, A_{1}, A_{2}, \phi\right) \Xi(\phi) e^{-i(n \psi+l \phi)} d \phi d \psi \\
\left.+\sum_{p_{m+2} n+q_{m+2} l=0} \int_{0}^{2 \pi} \int_{0}^{2 \pi} \hat{f}_{m+2}\left(\psi, A_{1}, A_{2}, \phi, z\right) \Xi(\phi) e^{-i(n \psi+l \phi)} \Pi_{2}(d z) d \phi d \psi\right], \\
\bar{\sigma}^{2}\left(A_{1}, A_{2}\right)=\bar{B}\left(A_{1}, A_{2}\right)=\frac{1}{4 \pi^{2}}\left[\sum_{j=1}^{m} \sum_{p_{j} n+q_{j} l=0} \int_{0}^{2 \pi} \int_{0}^{2 \pi} \hat{f}_{j}^{2}\left(\psi, A_{1}, A_{2}, \phi\right) \Xi(\phi) \Xi^{T}(\phi) e^{-i(n \psi+l \phi)} d \phi d \psi\right. \\
\left.\sum_{p_{m+1} n+q_{m+1} l=0} \int_{0}^{2 \pi} \int_{0}^{2 \pi} \int_{\mathbb{R}} \hat{f}_{m+1}^{2}\left(\psi, A_{1}, A_{2}, \phi, z\right) \Xi(\phi) \Xi^{T}(\phi) e^{-i(n \psi+l \phi)} \Pi_{1}(d z) d \phi d \psi\right] \\
\Xi(\phi)=\frac{1}{\omega\left(\eta^{2}+\omega^{2}\right)^{2}}\left(\begin{array}{c}
\left(\omega^{2}-\eta^{2}\right) \sin \phi-2 \eta \omega \cos \phi \\
-\left(\omega^{2}-\eta^{2}\right) \cos \phi-2 \eta \omega \sin \phi
\end{array}\right) \\
\hat{f}_{j}\left(\psi, A_{1}, A_{2}, \phi\right)=\tilde{f}_{j}\left(\psi, 0,0, A_{1}, A_{2}, \phi\right), j=\overline{0, m},
\end{gathered}
$$

$\Xi^{T}(\phi)$ is the vector transpose to the vector $\Xi(\phi), \bar{w}(t)=\left(\bar{w}_{i}(t), i=1,2\right), \bar{w}_{i}(t), i=1,2$ are independent onedimensional Wiener processes.

2. If $k<k_{0}$ then in the averaging equation (9) we must put $\tilde{f}_{0} \equiv 0$; if $k<2 k_{i}$ for some $i=\overline{1, m}$, then in the averaging equation (9) we must put $\tilde{f}_{i} \equiv 0$ for such $i$; if $k<2 k_{m+1}$ then in the averaging equation (9) we must put $\tilde{f}_{m+1} \equiv 0$; if $k<k_{m+2}$ then in the averaging equation (9) we must put $\tilde{f}_{m+2} \equiv 0$.

3. If $\mu_{j} \neq \frac{p_{j}}{q_{j}} \omega$ for some $j=\overline{0, m+2}$ and any relatively prime integers $p_{j}$ and $q_{j}$, then in averaging coefficients in (9) we must put $l=n=0$ in corresponding sums containing $\hat{f}_{j}$.

Proof. Let us make a change of variable $t \rightarrow t / \varepsilon^{k}$ at the system (8) and obtain for the process $\xi_{\varepsilon}(t)=$ $\left(N_{1}^{\varepsilon}(t), N_{2}^{\varepsilon}(t), A_{1}^{\varepsilon}(t), A_{2}^{\varepsilon}(t)\right)=\left(N_{1}\left(t / \varepsilon^{k}\right), N_{2}\left(t / \varepsilon^{k}\right), A_{1}\left(t / \varepsilon^{k}\right), A_{2}\left(t / \varepsilon^{k}\right)\right)$ the system of stochastic differential equations

$$
\begin{gathered}
d N_{1}^{\varepsilon}(t)=\left[-\frac{\eta_{1}}{\varepsilon^{k}} N_{1}^{\varepsilon}(t)+\frac{1}{\varepsilon^{k}} N_{2}^{\varepsilon}(t)\right] d t+\frac{2 \eta}{\left(\eta^{2}+\omega^{2}\right)^{2}} d H_{\varepsilon}(t), \\
d N_{2}^{\varepsilon}(t)=-\frac{\eta_{2}}{\varepsilon^{k}} N_{2}^{\varepsilon}(t) d t+\frac{1}{\left(\eta^{2}+\omega^{2}\right)} d H_{\varepsilon}(t), \\
d A_{1}^{\varepsilon}(t)=\Xi_{1}\left(\omega t / \varepsilon^{k}\right) d H_{\varepsilon}(t), \\
d A_{2}^{\varepsilon}(t)=\Xi_{2}\left(\omega t / \varepsilon^{k}\right) d H_{\varepsilon}(t), \\
d H_{\varepsilon}(t)=\left[\varepsilon^{k_{0}-k} \tilde{f}_{0}\left(\frac{\mu_{0} t}{\varepsilon^{k}}, \xi_{\varepsilon}(t), \frac{\omega t}{\varepsilon^{k}}\right)+\varepsilon^{k_{m+2}-k} \int_{\mathbb{R}} \tilde{f}_{m+2}\left(\frac{\mu_{m+2} t}{\varepsilon^{k}}, \xi_{\varepsilon}(t), \frac{\omega t}{\varepsilon^{k}}, z\right) \Pi_{2}(d z)\right] d t \\
+\sum_{i=1}^{m} \varepsilon^{k_{i}-k / 2} \tilde{f}_{i}\left(\frac{\mu_{i} t}{\varepsilon^{k}}, \xi_{\varepsilon}(t), \frac{\omega t}{\varepsilon^{k}}\right) d w_{i}^{\varepsilon}(t)+\varepsilon^{k_{m+1}} \int_{\mathbb{R}} \tilde{f}_{m+1}\left(\frac{\mu_{m+1} t}{\varepsilon^{k}}, \xi_{\varepsilon}(t), \frac{\omega t}{\varepsilon^{k}}, z\right) \tilde{\nu}_{1}^{\varepsilon}(d t, d z) \\
+\varepsilon^{k_{m+2}} \int_{\mathbb{R}} \tilde{f}_{m+2}\left(\frac{\mu_{m+2} t}{\varepsilon^{k}}, \xi_{\varepsilon}(t), \frac{\omega t}{\varepsilon^{k}}, z\right) \tilde{\nu}_{2}^{\varepsilon}(d t, d z),
\end{gathered}
$$

where $\Xi_{i}(\phi), i=1,2$ is a components of vector $\Xi(\phi), w_{i}^{\varepsilon}(t)=\varepsilon^{k / 2} w_{i}\left(t / \varepsilon^{k}\right), i=\overline{1, m}, \tilde{\nu}_{i}^{\varepsilon}(t, A)=\nu_{i}\left(t / \varepsilon^{k}, A\right)-$ $\Pi_{i}(A) t / \varepsilon^{k}, i=1,2$, here $A$ is a Borel set in $\mathbb{R}$. For each $\varepsilon>0$ the processes $w_{i}^{\varepsilon}(t), i=\overline{1, m}$ are independent onedimensional Wiener processes, and $\tilde{\nu}_{i}^{\varepsilon}(t, A), i=1,2$ are the independent centered Poisson measures independent on $w_{i}^{\varepsilon}(t), i=\overline{1, m}$. 
We have $N_{2}^{\varepsilon}(t)=\exp \left\{-\eta t / \varepsilon^{k}\right\} C_{2}\left(t / \varepsilon^{k}\right)$, and the process $C_{2}^{\varepsilon}(t)=C_{2}\left(t / \varepsilon^{k}\right)$ satisfies the stochastic differential equation

$$
d C_{2}^{\varepsilon}(t)=-\frac{e^{\eta t / \varepsilon^{k}}}{\eta^{2}+\omega^{2}} d H_{\varepsilon}(t)
$$

where $\left|C_{2}^{\varepsilon}(0)\right| \leq K$.

So, from boundedness of functions $f_{i}, i=\overline{0, m+2}$ and condition $\Pi_{i}(\mathbb{R})<\infty, i=1,2$ we have the estimate

$$
E\left|N_{2}^{\varepsilon}(t)\right|^{2} \leq K\left[e^{-\frac{2 \eta t}{\varepsilon^{k}}}+\varepsilon^{k}\left(1-e^{-\frac{2 \eta t}{\varepsilon^{k}}}\right)\left(t\left(\varepsilon^{2\left(k_{0}-k\right)}+\varepsilon^{2\left(k_{m+2}-k\right)}\right)+\sum_{i=1}^{m+2} \varepsilon^{2 k_{i}-k}\right)\right] .
$$

For the process $C_{\varepsilon}(t)=N_{1}^{\varepsilon}(t) e^{\eta t / \varepsilon^{k}}=C_{1}\left(t / \varepsilon^{k}\right)+C_{2}\left(t / \varepsilon^{k}\right) t / \varepsilon^{k}$, using the Ito formula, we obtain the stochastic differential equation

$$
C_{\varepsilon}(t)=C_{1}(0)+\frac{1}{\varepsilon^{k}} \int_{0}^{t} e^{\frac{\eta s}{\varepsilon^{k}}} N_{2}^{\varepsilon}(s) d s+\frac{2 \eta}{\left(\eta^{2}+\omega^{2}\right)^{2}} \int_{0}^{t} e^{\frac{\eta s}{\varepsilon^{k}}} d H_{\varepsilon}(s),
$$

then for the stochastic process $N_{1}^{\varepsilon}(t)$ we have

$$
N_{1}^{\varepsilon}(t)=e^{-\frac{\eta t}{\varepsilon^{k}}} C_{1}(0)+\frac{e^{-\frac{\eta t}{\varepsilon^{k}}}}{\varepsilon^{k}} \int_{0}^{t} e^{\frac{\eta s}{\varepsilon^{k}}} N_{2}^{\varepsilon}(s) d s+\frac{2 \eta e^{-\frac{\eta t}{\varepsilon^{k}}}}{\left(\eta^{2}+\omega^{2}\right)^{2}} \int_{0}^{t} e^{\frac{\eta s}{\varepsilon^{k}}} d H_{\varepsilon}(s) .
$$

From (10) we derive

$$
E\left|\frac{e^{-\frac{\eta t}{\varepsilon^{k}}}}{\varepsilon^{k}} \int_{0}^{t} e^{\frac{\eta s}{\varepsilon^{k}}} N_{2}^{\varepsilon}(s) d s\right| \leq K\left(\frac{t e^{-\frac{\eta t}{\varepsilon^{k}}}}{\varepsilon^{k}}+\frac{\varepsilon^{k / 2}}{\eta}(1+\sqrt{t})\left(1-e^{-\frac{\eta t}{\varepsilon^{k}}}\right)\right),
$$

and also we have the estimate

$$
E\left|\frac{2 \eta e^{-\frac{\eta t}{\varepsilon^{k}}}}{\left(\eta^{2}+\omega^{2}\right)^{2}} \int_{0}^{t} e^{\frac{\eta s}{\varepsilon^{k}}} d H_{\varepsilon}(s)\right| \leq K\left(\left(\varepsilon^{k_{0}}+\varepsilon^{k_{m+2}}\right)\left(1-e^{-\frac{\eta t}{\varepsilon^{k}}}\right)+\sum_{i=1}^{m+2} \varepsilon^{k_{i}} \sqrt{1-e^{-\frac{2 \eta t}{\varepsilon^{k}}}}\right) .
$$

Because $\left|C_{1}(0)\right| \leq K$, from (10), (11) and (12) we obtain $\lim _{\varepsilon \rightarrow 0} E\left|N_{2}^{\varepsilon}(t)\right|^{2}=0, \lim _{\varepsilon \rightarrow 0} E\left|N_{1}^{\varepsilon}(t)\right|=0$ and it is sufficient to study the behavior, as $\varepsilon \rightarrow 0$, of solution to the system of stochastic differential equations

$$
d A_{i}^{\varepsilon}(t)=\Xi_{i}\left(\frac{\omega t}{\varepsilon^{k}}\right) d \hat{H}_{\varepsilon}(t), i=1,2
$$

with initial conditions $A_{1}^{\varepsilon}(0)=A_{1}(0), A_{2}^{\varepsilon}(0)=A_{2}(0)$, where

$$
\begin{aligned}
& d \hat{H}_{\varepsilon}(t)=\left[\varepsilon^{k_{0}-k} \hat{f}_{0}\left(\frac{\mu_{0} t}{\varepsilon^{k}}, A_{1}^{\varepsilon}(t), A_{2}^{\varepsilon}(t), \frac{\omega t}{\varepsilon^{k}}\right)+\varepsilon^{k_{m+2}-k} \int_{\mathbb{R}} \hat{f}_{m+2}\left(\frac{\mu_{m+2} t}{\varepsilon^{k}}, A_{1}^{\varepsilon}(t), A_{2}^{\varepsilon}(t), \frac{\omega t}{\varepsilon^{k}}, z\right) \Pi_{2}(d z)\right] d t \\
& +\sum_{i=1}^{m} \varepsilon^{k_{i}-k / 2} \hat{f}_{i}\left(\frac{\mu_{i} t}{\varepsilon^{k}}, A_{1}^{\varepsilon}(t), A_{2}^{\varepsilon}(t), \frac{\omega t}{\varepsilon^{k}}\right) d w_{i}^{\varepsilon}(t)+\varepsilon^{k_{m+1}} \int_{\mathbb{R}} \hat{f}_{m+1}\left(\frac{\mu_{m+1} t}{\varepsilon^{k}}, A_{1}^{\varepsilon}(t), A_{2}^{\varepsilon}(t), \frac{\omega t}{\varepsilon^{k}}, z\right) \tilde{\nu}_{1}^{\varepsilon}(d t, d z) \\
& +\varepsilon^{k_{m+2}} \int_{\mathbb{R}} \hat{f}_{m+2}\left(\frac{\mu_{m+2} t}{\varepsilon^{k}}, A_{1}^{\varepsilon}(t), A_{2}^{\varepsilon}(t), \frac{\omega t}{\varepsilon^{k}}, z\right) \tilde{\nu}_{2}^{\varepsilon}(d t, d z), \hat{f}_{j}\left(\psi, A_{1}, A_{2}, \phi\right)=\tilde{f}_{j}\left(\psi, 0,0, A_{1}, A_{2}, \phi\right), j=\overline{0, m}, \\
& \hat{f}_{i}\left(\psi, A_{1}, A_{2}, \phi, z\right)=\tilde{f}_{i}\left(\psi, 0,0, A_{1}, A_{2}, \phi, z\right), i=m+1, m+2 .
\end{aligned}
$$

Let us denote $A_{\varepsilon}(t)=\left(A_{1}^{\varepsilon}(t), A_{2}^{\varepsilon}(t)\right)$. Using conditions on coefficients of equation (13) and properties of stochastic integrals we obtain estimates

$$
E\left\|A_{\varepsilon}(t)\right\|^{2} \leq K\left[1+t^{2}\left(\varepsilon^{2\left(k_{0}-k\right)}+\varepsilon^{2\left(k_{m+2}-k\right)}\right)+t \sum_{i=1}^{m+2} \varepsilon^{2 k_{i}-k}\right],
$$




$$
E \| A_{\varepsilon}(t)-\left.A_{\varepsilon}(s)\right|^{2} \leq K\left[|t-s|^{2}\left(\varepsilon^{2\left(k_{0}-k\right)}+\varepsilon^{2\left(k_{m+2}-k\right)}\right)+|t-s| \sum_{i=1}^{m+2} \varepsilon^{2 k_{i}-k}\right] .
$$

Similarly for the process $\zeta_{\varepsilon}(t)=\left(\zeta_{\varepsilon}^{(1)}(t), \zeta_{\varepsilon}^{(2)}(t)\right)$, where

$$
\begin{gathered}
\zeta_{\varepsilon}^{(i)}(t)=\int_{0}^{t} \Xi_{i}\left(\frac{\omega s}{\varepsilon^{k}}\right) d M_{\varepsilon}(s), i=1,2, \\
d M_{\varepsilon}(t)=\sum_{i=1}^{m} \varepsilon^{k_{i}-k / 2} \hat{f}_{i}\left(\frac{\mu_{i} t}{\varepsilon^{k}}, A_{1}^{\varepsilon}(t), A_{2}^{\varepsilon}(t), \frac{\omega t}{\varepsilon^{k}}\right) d w_{i}^{\varepsilon}(t)+\int_{\mathbb{R}} \hat{f}_{m+1}\left(\frac{\mu_{m+1} t}{\varepsilon^{k}}, A_{1}^{\varepsilon}(t), A_{2}^{\varepsilon}(t), \frac{\omega t}{\varepsilon^{k}}, z\right) \tilde{\nu}_{1}^{\varepsilon}(d t, d z) \\
+\varepsilon^{k_{m+2}} \int_{\mathbb{R}} \hat{f}_{m+2}\left(\frac{\mu_{m+2} t}{\varepsilon^{k}}, A_{1}^{\varepsilon}(t), A_{2}^{\varepsilon}(t), \frac{\omega t}{\varepsilon^{k}}, z\right) \tilde{\nu}_{2}^{\varepsilon}(d t, d z),
\end{gathered}
$$

we derive estimates

$$
E\left\|\zeta_{\varepsilon}(t)\right\|^{2} \leq K t \sum_{i=1}^{m+2} \varepsilon^{2 k_{i}-k}, \quad E|| \zeta_{\varepsilon}(t)-\zeta_{\varepsilon}(s) \|^{2} \leq K|t-s| \sum_{i=1}^{m+2} \varepsilon^{2 k_{i}-k} .
$$

Therefore for stochastic process $\eta_{\varepsilon}(t)=\left(A_{\varepsilon}(t), \zeta_{\varepsilon}(t)\right)$ conditions of weak compactness [20] are fulfilled:

$$
\lim _{h \downarrow 0} \varlimsup_{\varepsilon \rightarrow 0} \sup _{|t-s|<h} \mathrm{P}\left\{\left|\eta_{\varepsilon}(t)-\eta_{\varepsilon}(s)\right|>\delta\right\}=0
$$

for any $\delta>0, t, s \in[0, T]$,

$$
\lim _{N \rightarrow \infty} \varlimsup_{\varepsilon \rightarrow 0} \sup _{t \in[0, T]} \mathrm{P}\left\{\left|\eta_{\varepsilon}(t)\right|>N\right\}=0 .
$$

So for any sequence $\varepsilon_{n} \rightarrow 0, n=1,2, \ldots$ there exists a subsequence $\varepsilon_{m}=\varepsilon_{n(m)} \rightarrow 0, m=1,2, \ldots$, probability space, stochastic processes $\bar{A}_{\varepsilon_{m}}(t)=\left(\bar{A}_{1}^{\varepsilon_{m}}(t), \bar{A}_{2}^{\varepsilon_{m}}(t)\right), \bar{\zeta}_{\varepsilon_{m}}(t), \bar{A}(t)=\left(\bar{A}_{1}(t), \bar{A}_{2}(t)\right), \bar{\zeta}(t)$ defined on this space, such that $\bar{A}_{\varepsilon_{m}}(t) \rightarrow \bar{A}(t), \bar{\zeta}_{\varepsilon_{m}}(t) \rightarrow \bar{\zeta}(t)$ in probability, as $\varepsilon_{m} \rightarrow 0$, and finite-dimensional distributions of $\bar{A}_{\varepsilon_{m}}(t), \bar{\zeta}_{\varepsilon_{m}}(t)$ are coincide with finite-dimensional distributions of $A_{\varepsilon_{m}}(t), \zeta_{\varepsilon_{m}}(t)$. Since we are interested in limit behaviour of distributions, we can consider processes $A_{\varepsilon_{m}}(t)$, and $\zeta_{\varepsilon_{m}}(t)$ instead of $\bar{A}_{\varepsilon_{m}}(t), \bar{\zeta}_{\varepsilon_{m}}(t)$.

From (13) we obtain equation

$$
A_{\varepsilon_{m}}(t)=A(0)+\int_{0}^{t} \alpha_{\varepsilon_{m}}\left(s, A_{\varepsilon_{m}}(s)\right) d s+\zeta_{\varepsilon_{m}}(t), \quad A(0)=\left(A_{1}(0), A_{2}(0)\right),
$$

where $\alpha_{\varepsilon}(t, A)=\left(\alpha_{\varepsilon}^{(1)}\left(t, A_{1}, A_{2}\right), \alpha_{\varepsilon}^{(2)}\left(t, A_{1}, A_{2}\right)\right)$,

$\alpha_{\varepsilon}^{(i)}\left(t, A_{1}, A_{2}\right)=\Xi_{i}\left(\frac{\omega t}{\varepsilon^{k}}\right)\left[\varepsilon^{k_{0}-k} \hat{f}_{0}\left(\frac{\mu_{0} t}{\varepsilon^{k}}, A_{1}, A_{2}, \frac{\omega t}{\varepsilon^{k}}\right)+\varepsilon^{k_{m+2}-k} \int_{\mathbb{R}} \hat{f}_{m+2}\left(\frac{\mu_{m+2} t}{\varepsilon^{k}}, A_{1}, A_{2}, \frac{\omega t}{\varepsilon^{k}}, z\right) \Pi_{2}(d z)\right]$,

$i=1,2$.

It should be noted that process $\zeta_{\varepsilon}(t)$ is the vector-valued square integrable martingale with matrix characteristic

$$
\begin{aligned}
& \left\langle\zeta_{\varepsilon}^{(l)}, \zeta_{\varepsilon}^{(n)}\right\rangle(t)=\sum_{j=1}^{m} \int_{0}^{t} \sigma_{\varepsilon}^{(l, j)}\left(s, A_{1}^{\varepsilon}(s), A_{2}^{\varepsilon}(s)\right) \sigma_{\varepsilon}^{(n, j)}\left(s, A_{1}^{\varepsilon}(s), A_{2}^{\varepsilon}(s)\right) d s \\
& +\frac{1}{\varepsilon^{k}} \int_{0}^{t} \int_{\mathbb{R}} \gamma_{\varepsilon}^{(l)}\left(s, A_{1}^{\varepsilon}(s), A_{2}^{\varepsilon}(s), z\right) \gamma_{\varepsilon}^{(n)}\left(s, A_{1}^{\varepsilon}(s), A_{2}^{\varepsilon}(s), z\right) \Pi_{1}(d z) d s \\
& +\frac{1}{\varepsilon^{k}} \int_{0}^{t} \int_{\mathbb{R}} \delta_{\varepsilon}^{(l)}\left(s, A_{1}^{\varepsilon}(s), A_{2}^{\varepsilon}(s), z\right) \delta_{\varepsilon}^{(n)}\left(s, A_{1}^{\varepsilon}(s), A_{2}^{\varepsilon}(s), z\right) \Pi_{2}(d z) d s, l, n=1,2,
\end{aligned}
$$


where

$$
\begin{aligned}
& \sigma_{\varepsilon}^{(l, j)}\left(s, A_{1}, A_{2}\right)=\varepsilon^{k_{j}-k / 2} \Xi_{l}\left(\frac{\omega s}{\varepsilon^{k}}\right) \hat{f}_{j}\left(\frac{\mu_{j} s}{\varepsilon^{k}}, A_{1}, A_{2}, \frac{\omega s}{\varepsilon^{k}}\right), \\
& \gamma_{\varepsilon}^{(l)}\left(s, A_{1}, A_{2}, z\right)=\varepsilon^{k_{m+1}} \Xi_{l}\left(\frac{\omega s}{\varepsilon^{k}}\right) \hat{f}_{m+1}\left(\frac{\mu_{m+1} s}{\varepsilon^{k}}, A_{1}, A_{2}, \frac{\omega s}{\varepsilon^{k}}, z\right), \\
& \delta_{\varepsilon}^{(l)}\left(s, A_{1}, A_{2}, z\right)=\varepsilon^{k_{m+2}} \Xi_{l}\left(\frac{\omega s}{\varepsilon^{k}}\right) \hat{f}_{m+2}\left(\frac{\mu_{m+2} s}{\varepsilon^{k}}, A_{1}, A_{2}, \frac{\omega s}{\varepsilon^{k}}, z\right), \quad l=1,2 .
\end{aligned}
$$

For processes $A_{\varepsilon}(t)$ and $\zeta_{\varepsilon}(t)$ following estimates hold true

$$
\begin{gathered}
E\left\|A_{\varepsilon}(t)-A_{\varepsilon}(s)\right\|^{4} \leq K\left[\left(\varepsilon^{4\left(k_{0}-k\right)}+\varepsilon^{4\left(k_{m+2}-k\right)}\right)|t-s|^{4}+E\left\|\zeta_{\varepsilon}(t)-\zeta_{\varepsilon}(s)\right\|^{4}\right] \\
E\left\|\zeta_{\varepsilon}(t)-\zeta_{\varepsilon}(s)\right\|^{4} \leq K\left[\sum_{j=1}^{m+2} \varepsilon^{4 k_{j}-2 k}|t-s|^{2}+\left(\varepsilon^{4 k_{m+1}-3 k / 2}+\varepsilon^{4 k_{m+2}-3 k / 2}\right)|t-s|^{3 / 2}\right. \\
\left.+\left(\varepsilon^{4 k_{m+1}-k}+\varepsilon^{4 k_{m+2}-k}\right)|t-s|\right], \\
E\left\|A_{\varepsilon}(t)-A_{\varepsilon}(s)\right\|^{8} \leq K, \quad E\left\|\zeta_{\varepsilon}(t)-\zeta_{\varepsilon}(s)\right\|^{8} \leq K .
\end{gathered}
$$

Here we used the estimate for stochastic integrals with respect to Wiener process:

$$
E\left[\int_{s}^{t} f(\tau) d w(\tau)\right]^{2 n} \leq[n(2 n-1)]^{n}|t-s|^{n-1} E\left[\int_{s}^{t} f^{2 n}(\tau) d \tau\right], n \geq 1 .
$$

For estimating of stochastic integrals with respect to centered Poisson measures we used the Ito formula for the process

$$
\left(\int_{0}^{t} \int_{\mathbb{R}} \varepsilon^{k_{m+i}} \hat{f}_{m+i} \tilde{\nu}_{i}^{\varepsilon}(d \tau, d z)\right)^{4 n}, \quad i=1,2, \quad n=1,2
$$

Since $A_{\varepsilon_{m}}(t) \rightarrow \bar{A}(t), \zeta_{\varepsilon_{m}}(t) \rightarrow \bar{\zeta}(t)$ in probability, as $\varepsilon_{m} \rightarrow 0$, then, using (17), from (15) and (16) we obtain estimates

$$
\begin{aligned}
& E\|\bar{A}(t)-\bar{A}(s)\|^{4} \leq K\left(|t-s|^{4}+|t-s|^{2}\right), \\
& E\|\bar{\zeta}(t)-\bar{\zeta}(s)\|^{4} \leq C|t-s|^{2} .
\end{aligned}
$$

Therefore processes $\bar{A}(t)$ and $\bar{\zeta}(t)$ satisfy the Kolmogorov's continuity condition [21].

Let us consider the case $k_{0}=2 k_{i}=k_{m+2}, i=\overline{1, m+1}$ and $\mu_{i}=p_{i} \omega / q_{i}$ for all $i=\overline{0, m+2}$, where $p_{i}$ and $q_{i}$ are some relatively prime integers. Under these conditions from Fourier series expansion of integrand functions on variables $\mu_{i} t / \varepsilon^{k}, i=\overline{0, m+2}$ and $\omega t / \varepsilon^{k}$ in corresponding terms, we obtain for $l, n=1,2$

$$
\begin{aligned}
& \lim _{\varepsilon \rightarrow 0} \frac{1}{t} \int_{0}^{t} \alpha_{\varepsilon}^{(l)}\left(s, A_{1}, A_{2}\right) d s=\bar{\alpha}_{l}\left(A_{1}, A_{2}\right), \\
& \lim _{\varepsilon \rightarrow 0} \frac{1}{t} \int_{0}^{t}\left[\sum_{j=1}^{m} \sigma_{\varepsilon}^{(l, j)}\left(s, A_{1}, A_{2}\right) \sigma_{\varepsilon}^{(n, j)}\left(s, A_{1}, A_{2}\right)+\frac{1}{\varepsilon^{k}} \int_{R} \gamma_{\varepsilon}^{(l)}\left(s, A_{1}, A_{2}, z\right) \gamma_{\varepsilon}^{(n)}\left(s, A_{1}, A_{2}, z\right) \Pi_{1}(d z)\right. \\
& \left.+\frac{1}{\varepsilon^{k}} \int_{R} \delta_{\varepsilon}^{(l)}\left(s, A_{1}, A_{2}, z\right) \delta_{\varepsilon}^{(n)}\left(s, A_{1}, A_{2}, z\right) \Pi_{2}(d z)\right] d s=\bar{B}_{l n}\left(A_{1}, A_{2}\right),
\end{aligned}
$$

where functions $\bar{\alpha}_{l}\left(A_{1}, A_{2}\right), l=1,2$ and $\bar{B}\left(A_{1}, A_{2}\right)=\left\{\bar{B}_{l n}\left(A_{1}, A_{2}\right), l, n=1,2\right\}$ are defined in the conditions of theorem. 
Since $A_{\varepsilon_{m}}(t) \rightarrow \bar{A}(t), \zeta_{\varepsilon_{m}}(t) \rightarrow \bar{\zeta}(t)$ in probability, as $\varepsilon_{m} \rightarrow 0$, processes $\bar{A}(t), \bar{\zeta}(t)$ are continuous, functions $f_{j}, j=\overline{0, m+2}$ bounded and satisfy Lipschitz condition on $y_{i}, i=\overline{1,4}$, function $\Xi(\phi)$ is bounded, then from Lemma 1, Remark 1 and relationships (14), (18) it follows

$$
\bar{A}(t)=A(0)+\int_{0}^{t} \bar{\alpha}\left(\bar{A}_{1}(s), \bar{A}_{2}(s)\right) d s+\bar{\zeta}(t), \quad A(0)=\left(A_{1}(0), A_{2}(0)\right),
$$

almost surely, where $\bar{\zeta}(t)=\left(\bar{\zeta}^{(1)}(t), \bar{\zeta}^{(2)}(t)\right)$ is continuous vector-valued martingale with matrix characteristic

$$
\left\langle\bar{\zeta}^{(i)}, \bar{\zeta}^{(j)}\right\rangle(t)=\int_{0}^{t} \bar{B}_{i j}\left(\bar{A}_{1}(s), \bar{A}_{2}(s)\right) d s, \quad i, j=1,2 .
$$

Hence [22] there exists Wiener process $\bar{w}(t)=\left(\bar{w}_{i}(t), i=1,2\right)$, such that

$$
\bar{\zeta}(t)=\int_{0}^{t} \bar{\sigma}\left(\bar{A}_{1}(s), \bar{A}_{2}(s)\right) d \bar{w}(s), \quad \bar{\sigma}\left(A_{1}, A_{2}\right)=\left\{\bar{B}\left(A_{1}, A_{2}\right)\right\}^{1 / 2} .
$$

Relationships (19), (20) mean that process $\bar{A}(t)$ satisfies equation (9). Under conditions of theorem the equation (9) has unique solution. Therefore process $\bar{A}(t)$ does not depend on choosing of sub-sequence $\varepsilon_{m} \rightarrow 0$, and finitedimensional distributions of process $A_{\varepsilon_{m}}(t)$ converge to finite-dimensional distributions of process $\bar{A}(t)$. Since processes $A_{\varepsilon_{m}}(t)$ and $\bar{A}(t)$ are Markov processes then using the conditions for weak convergence of Markov processes [21] we finish the proof of statement 1) of the theorem.

Let us consider the cases $k<k_{1}$ or $k<k_{m+2}$. Then the corresponding terms in the coefficients $\alpha_{\varepsilon}^{(i)}\left(t, A_{1}, A_{2}\right)$, $i=1,2$ of equation (14) tend to zero, as $\varepsilon \rightarrow 0$.

In the case $k<2 k_{i}$ for some $i=\overline{1, m}$, we have in (18)

$$
\sigma_{\varepsilon}^{(l, i)}\left(s, A_{1}^{\varepsilon}, A_{2}^{\varepsilon}\right) \sigma_{\varepsilon}^{(n, i)}\left(s, A_{1}^{\varepsilon}, A_{2}^{\varepsilon}\right)=O\left(\varepsilon^{2 k_{i}-k}\right), \quad l, n=1,2
$$

for such $i=\overline{1, m}$.

In the case $k<2 k_{m+1}$ we have in (18)

$$
\frac{1}{\varepsilon^{k}} \int_{R} \gamma_{\varepsilon}^{(l)}\left(s, A_{1}, A_{2}, z\right) \gamma_{\varepsilon}^{(n)}\left(s, A_{1}, A_{2}, z\right) \Pi_{1}(d z)=O\left(\varepsilon^{2 k_{m+1}-k}\right), \quad l, n=1,2 .
$$

In all cases we have $k<2 k_{m+2}$, and therefore

$$
\lim _{\varepsilon \rightarrow 0} \frac{1}{\varepsilon^{k}} \int_{R} \delta_{\varepsilon}^{(l)}\left(s, A_{1}, A_{2}, z\right) \delta_{\varepsilon}^{(n)}\left(s, A_{1}, A_{2}, z\right) \Pi_{2}(d z)=0, \quad l, n=1,2 .
$$

If $\mu_{j} \neq \frac{p_{j}}{q_{j}} \omega$ for some $j=\overline{0, m+2}$ and any relatively prime integers $p_{j}$ and $q_{j}$, then in (18) we obtain in corresponding averaged coefficient only one term for $n=l=0$, instead of sum over all $n, l$ such that $p_{j} n+q_{j} l=0$.

Repeating with obvious modifications the proof of statement 1 ) of theorem we obtain proof of the statements 2 ) and 3 ).

\section{The case of two pairs of imaginary adjoined roots of characteristic equation}

In this section we will study the following case:

$$
b_{1}=0, b_{3}=0, b_{2}>0, b_{4}>0, b_{2}^{2}>4 b_{4} .
$$


Characteristic equation has a roots

$$
\lambda_{1,2}= \pm i \omega_{1}, \lambda_{3,4}= \pm i \omega_{2}, \text { where } \omega_{1}^{2}=\frac{1}{2}\left(b_{2}+\sqrt{b_{2}^{2}-4 b_{4}}\right), \omega_{2}^{2}=\frac{1}{2}\left(b_{2}-\sqrt{b_{2}^{2}-4 b_{4}}\right) .
$$

If $\varepsilon=0$ then the equation (1) has general solution in the form

$$
x(t)=A_{11} \cos \omega_{1} t+A_{12} \sin \omega_{1} t+A_{21} \cos \omega_{2} t+A_{22} \sin \omega_{2} t .
$$

Let us denote

$$
A(t)=\left(A_{11}(t), A_{12}(t), A_{21}(t), A_{22}(t)\right), \quad \Phi(t)=\left(\cos \omega_{1} t, \sin \omega_{1} t, \cos \omega_{2} t, \sin \omega_{2} t\right)
$$

and let us consider the following representation of the solution $\mathbf{y}(t)$ to the system (2):

$$
y_{i}(t)=\left(A(t) \cdot \frac{d^{i-1}}{d t^{i-1}} \Phi(t)\right), i=\overline{1,4} .
$$

We can solve the system of linear equations (21) with respect to $\left(A_{11}(t), A_{12}(t), A_{21}(t), A_{22}(t)\right)$ and using the Ito formula we derive the system of stochastic differential equations:

$$
d A(t)=\Theta\left(\omega_{1} t, \omega_{2} t\right) d H(t)
$$

where

$$
\begin{gathered}
\Theta\left(\phi_{1}, \phi_{2}\right)=\frac{1}{\omega_{1}^{2}-\omega_{2}^{2}}\left(\frac{\sin \phi_{1}}{\omega_{1}},-\frac{\cos \phi_{1}}{\omega_{1}},-\frac{\sin \phi_{2}}{\omega_{2}}, \frac{\cos \phi_{2}}{\omega_{2}}\right), \\
d H(t)=\left[\varepsilon^{k_{0}} \tilde{f}_{0}\left(\mu_{0} t, A(t), \omega_{1} t, \omega_{2} t\right)+\varepsilon^{k_{m+2}} \int_{\mathbb{R}} \tilde{f}_{m+2}\left(\mu_{m+2} t, A(t), \omega_{1} t, \omega_{2} t, z\right) \Pi_{2}(d z)\right] d t \\
+\sum_{i=1}^{m} \varepsilon^{k_{i}} \tilde{f}_{i}\left(\mu_{i} t, A(t), \omega_{1} t, \omega_{2} t\right) d \omega_{i}(t)+\varepsilon^{k_{m+1}} \int_{\mathbb{R}} \tilde{f}_{m+1}\left(\mu_{m+1} t, A(t), \omega_{1} t, \omega_{2} t, z\right) \tilde{\nu}_{1}(d t, d z) \\
+\varepsilon^{k_{m+2}} \int_{\mathbb{R}} \tilde{f}_{m+2}\left(\mu_{m+2} t, A(t), \omega_{1} t, \omega_{2} t, z\right) \tilde{\nu}_{2}(d t, d z),
\end{gathered}
$$

where $\tilde{f}_{i}\left(\mu_{i} t, A(t), \omega_{1} t, \omega_{2} t\right), i=\overline{0, m}$ are obtained from $f_{i}\left(\mu_{i} t, \mathbf{y}(t)\right), i=\overline{0, m}$ and $\tilde{f}_{i}\left(\mu_{i} t, A(t), \omega_{1} t, \omega_{2} t, z\right), i=$ $m+1, m+2$ are obtained from $f_{i}\left(\mu_{i} t, \mathbf{y}(t), z\right), i=m+1, m+2$ using $(21)$.

\section{Theorem 4}

Let $\Pi_{i}(\mathbb{R})<\infty, i=1,2, t \in\left[0, t_{0}\right], k=\min \left(k_{0}, 2 k_{1}, \ldots, 2 k_{m+1}, k_{m+2}\right)$. Let us suppose, that functions $f_{j}, j=$ $\overline{0, m+2}$ bounded and satisfy Lipschitz condition on $y_{i}, i=\overline{1,4}$. If given below matrix $\bar{\sigma}^{2}\left(A_{1}, A_{2}\right)$ is non-negative definite, then:

1. Let $\mu_{j}=\frac{p_{j}^{(1)}}{q_{j}^{(1)}} \omega_{1}=\frac{p_{j}^{(2)}}{q_{j}^{(2)}} \omega_{2}$ for all $j=\overline{0, m+2}$, where $p_{j}^{(i)}$ and $q_{j}^{(i)}$ are some relatively prime integers, $i=1,2$, $j=\overline{0, m+2}$. If $k_{0}=2 k_{i}=k_{m+2}, i=\overline{1, m+1}$, then the stochastic process $A_{\varepsilon}(t)=A\left(t / \varepsilon^{k}\right)$ weakly converges, as $\varepsilon \rightarrow 0$, to the stochastic process $\bar{A}(t)=\left(\bar{A}_{11}(t), \bar{A}_{12}(t), \bar{A}_{21}(t), \bar{A}_{22}(t)\right)$ which is the solution to the system of stochastic differential equations

$$
d \bar{A}(t)=\bar{\alpha}(\bar{A}(t)) d t+\bar{\sigma}(\bar{A}(t)) d \bar{w}(t), \quad \bar{A}(0)=\left(A_{11}(0), A_{12}(0), A_{21}(0), A_{22}(0)\right),
$$

where

$$
\begin{aligned}
& \bar{\alpha}(A)=\frac{1}{8 \pi^{3}}\left[\sum_{\sigma_{0}} \int_{0}^{2 \pi} \int_{0}^{2 \pi} \int_{0}^{2 \pi} \hat{f}_{0}\left(\psi, A, \phi_{1}, \phi_{2}\right) \Theta\left(\phi_{1}, \phi_{2}\right) e^{-i\left(n_{1} \phi_{1}+n_{2} \phi_{2}+n_{3} \psi\right)} d \phi_{1} d \phi_{2} d \psi\right. \\
& \left.+\sum_{\sigma_{m+2}} \int_{0}^{2 \pi} \int_{0}^{2 \pi} \int_{0}^{2 \pi} \int_{\mathbb{R}} \hat{f}_{m+2}\left(\psi, A, \phi_{1}, \phi_{2}, z\right) \Theta\left(\phi_{1}, \phi_{2}\right) e^{\left.-i\left(n_{1} \phi_{1}+n_{2} \phi_{2}+n_{3} \psi\right)\right)} \Pi_{2}(d z) d \phi_{1} d \phi_{2} d \psi\right],
\end{aligned}
$$




$$
\begin{aligned}
& \bar{\sigma}^{2}(A)=\bar{B}(A)=\frac{1}{8 \pi^{3}}\left[\sum_{j=1}^{m} \sum_{\sigma_{j}} \int_{0}^{2 \pi} \int_{0}^{2 \pi} \int_{0}^{2 \pi} \hat{f}_{j}^{2}\left(\psi, A, \phi_{1}, \phi_{2}\right) \Theta^{T}\left(\phi_{1}, \phi_{2}\right) \Theta\left(\phi_{1}, \phi_{2}\right) e^{-i\left(n_{1} \phi_{1}+n_{2} \phi_{2}+n_{3} \psi\right)} d \phi_{1} d \phi_{2} d \psi\right. \\
& \left.+\sum_{\sigma_{m+1}} \int_{0}^{2 \pi} \int_{0}^{2 \pi} \int_{0}^{2 \pi} \int_{\mathbb{R}} \hat{f}_{m+1}^{2}\left(\psi, A, \phi_{1}, \phi_{2}, z\right) \Theta^{T}\left(\phi_{1}, \phi_{2}\right) \Theta\left(\phi_{1}, \phi_{2}\right) e^{-i\left(n_{1} \phi_{1}+n_{2} \phi_{2}+n_{3} \psi\right)} \Pi_{1}(d z) d \phi_{1} d \phi_{1} d \psi\right],
\end{aligned}
$$

where $\sum_{\sigma_{j}}$ means summation over all negative, positive and equal zero integers $n_{1}, n_{2}, n_{3}$ such, that $\quad n_{1} p_{j}^{(2)} q_{j}^{(1)}+n_{2} p_{j}^{(1)} q_{j}^{(2)}+n_{3} p_{j}^{(1)} p_{j}^{(2)}=0, \quad j=\overline{0, m+2} ; \quad A=\left(A_{11}, A_{12}, A_{21}, A_{22}\right) ; \quad \hat{f}_{j}\left(\psi, A, \phi_{1}, \phi_{2}\right)=$ $\tilde{f}_{j}\left(\psi, 0,0, A, \phi_{1}, \phi_{2}\right), j=\overline{0, m}, \hat{f}_{i}\left(\psi, A, \phi_{1}, \phi_{2}, z\right)=\tilde{f}_{i}\left(\psi, 0,0, A, \phi_{1}, \phi_{2}, z\right), i=m+1, m+2 ; \Theta^{T}\left(\phi_{1}, \phi_{2}\right)$ is the vector transpose to the vector $\Theta\left(\phi_{1}, \phi_{2}\right) ; \bar{w}(t)=\left(\bar{w}_{i}(t), i=\overline{1,4}\right), \bar{w}_{i}(t), i=\overline{1,4}$ are independent one-dimensional Wiener processes.

2. Let $k_{0}=2 k_{i}=k_{m+2}, i=\overline{1, m+1}$. If $\mu_{j}=\frac{p_{j}^{(1)}}{q_{j}^{(1)}} \omega_{1}$ for some $j=\overline{0, m+2}$, where $p_{j}^{(1)}, q_{j}^{(1)}$ are some relatively prime integers, and $\mu_{j} \neq \frac{p_{j}^{(2)}}{q_{j}^{(2)}} \omega_{2}$ for any relatively prime integers $p_{j}^{(2)}$ and $q_{j}^{(2)}$, then for such $j$ we must put $n_{2}=0$ in sum $\sum_{\sigma_{j}}$ and take summation over all $n_{1}$ and $n_{3}$ such, that $n_{1} q_{j}^{(1)}+n_{3} p_{j}^{(1)}=0$. If $\mu_{j}=\frac{p_{j}^{(2)}}{q_{j}^{(2)}} \omega_{2}$ for some $j=\overline{0, m+2}$, where $p_{j}^{(2)}, q_{j}^{(2)}$ are some relatively prime integers, and $\mu_{j} \neq \frac{p_{j}^{(1)}}{q_{j}^{(1)}} \omega_{1}$ for any relatively prime integers $p_{j}^{(1)}$ and $q_{j}^{(1)}$, then for such $j$ we must put $n_{1}=0$ in sum $\sum_{\sigma_{j}}$ and take summation over all $n_{2}$ and $n_{3}$ such, that $n_{2} q_{j}^{(2)}+n_{3} p_{j}^{(2)}=0$.

3. Let $k_{0}=2 k_{i}=k_{m+2}, i=\overline{1, m+1}$. If $\mu_{j} \neq \frac{p_{j}^{(i)}}{q_{j}^{(i)}} \omega_{i}, i=1,2$ for some $j=\overline{0, m+2}$, where $p_{j}^{(i)}, q_{j}^{(i)}, i=1,2$ are any relatively prime integers, and $\omega_{1}=\frac{p}{q} \omega_{2}$ for some relatively prime integers $p$ and $q$, then for such $j$ we must put $n_{3}=0$ in sum $\sum_{\sigma_{j}}$ and take summation over all $n_{1}$ and $n_{2}$ such, that $n_{1} q+n_{2} p=0$.

4. Let $k_{0}=2 k_{i}=k_{m+2}, i=\overline{1, m+1}$. If $\mu_{j} \neq \frac{p_{j}^{(i)}}{q_{j}^{(i)}} \omega_{i}, i=1,2$ for some $j=\overline{0, m+2}$, where $p_{j}^{(i)}, q_{j}^{(i)}, i=1,2$ are any relatively prime integers, and $\omega_{1} \neq \frac{p}{q} \omega_{2}$ for any relatively prime integers $p$ and $q$, then for such $j$ we must put $n_{1}=n_{2}=n_{3}=0$ in $\operatorname{sum} \sum_{\sigma_{j}}$.

5. If $k<k_{0}$ then in the averaging equation (23) we must put $\tilde{f}_{0} \equiv 0$; if $k<2 k_{i}$ for some $i=\overline{1, m}$, then in the averaging equation (23) we must put $\tilde{f}_{i} \equiv 0$ for such $i$; if $k<2 k_{m+1}$ then in the averaging equation (23) we must put $\tilde{f}_{m+1} \equiv 0$; if $k<k_{m+2}$ then in the averaging equation (23) we must put $\tilde{f}_{m+2} \equiv 0$.

Proof. Let us make a change of variable $t \rightarrow t / \varepsilon^{k}$ at the system (22) and obtain for the process $A_{\varepsilon}(t)=$ $\left(A_{11}\left(t / \varepsilon^{k}\right), A_{12}\left(t / \varepsilon^{k}\right), A_{21}\left(t / \varepsilon^{k}\right), A_{22}\left(t / \varepsilon^{k}\right)\right)$ the system of stochastic differential equations

$$
d A_{\varepsilon}(t)=\Theta\left(\omega_{1} t / \varepsilon^{k}, \omega_{2} t / \varepsilon^{k}\right) d H_{\varepsilon}(t),
$$

where

$$
\begin{aligned}
& d H_{\varepsilon}(t)=\left[\varepsilon^{k_{0}-k} \tilde{f}_{0}\left(\frac{\mu_{0} t}{\varepsilon^{k}}, A_{\varepsilon}(t), \frac{\omega_{1} t}{\varepsilon^{k}}, \frac{\omega_{2} t}{\varepsilon^{k}}\right)+\varepsilon^{k_{m+2}-k} \int_{\mathbb{R}} \tilde{f}_{m+2}\left(\frac{\mu_{m+2} t}{\varepsilon^{k}}, A_{\varepsilon}(t), \frac{\omega_{1} t}{\varepsilon^{k}}, \frac{\omega_{2} t}{\varepsilon^{k}}, z\right) \Pi_{2}(d z)\right] d t \\
& +\sum_{i=1}^{m} \varepsilon^{k_{i}-k / 2} \tilde{f}_{i}\left(\frac{\mu_{i} t}{\varepsilon^{k}}, A_{\varepsilon}(t), \frac{\omega_{1} t}{\varepsilon^{k}}, \frac{\omega_{2} t}{\varepsilon^{k}}\right) d w_{i}^{\varepsilon}(t)+\varepsilon^{k_{m+1}} \int_{\mathbb{R}} \tilde{f}_{m+1}\left(\frac{\mu_{m+1} t}{\varepsilon^{k}}, A_{\varepsilon}(t), \frac{\omega_{1} t}{\varepsilon^{k}}, \frac{\omega_{2} t}{\varepsilon^{k}}, z\right) \tilde{\nu}_{1}^{\varepsilon}(d t, d z) \\
& +\varepsilon^{k_{m+2}} \int_{\mathbb{R}} \tilde{f}_{m+2}\left(\frac{\mu_{m+2} t}{\varepsilon^{k}}, A_{\varepsilon}(t), \frac{\omega_{1} t}{\varepsilon^{k}}, \frac{\omega_{2} t}{\varepsilon^{k}}, z\right) \tilde{\nu}_{2}^{\varepsilon}(d t, d z),
\end{aligned}
$$

where $w_{i}^{\varepsilon}(t)=\varepsilon^{k / 2} w_{i}\left(t / \varepsilon^{k}\right), i=\overline{1, m}, \tilde{\nu}_{i}^{\varepsilon}(t, A)=\nu_{i}\left(t / \varepsilon^{k}, A\right)-\Pi_{i}(A) t / \varepsilon^{k}, i=1,2$, here $A$ is a Borel set in $\mathbb{R}$. For each $\varepsilon>0$ the processes $w_{i}^{\varepsilon}(t), i=\overline{1, m}$ are independent one-dimensional Wiener processes, and $\tilde{\nu}_{i}^{\varepsilon}(t, A), i=$ 1,2 are independent centered Poisson measures, which are independent on $w_{i}^{\varepsilon}(t), i=\overline{1, m}$. 
We can apply the same arguments as in a proof of Theorem 3 to the processes $A_{\varepsilon}(t)$ and $\zeta_{\varepsilon}(t)=\left(\zeta_{\varepsilon}^{(i)}(t)\right.$, $i=\overline{1,4})$, where

$$
\begin{gathered}
\zeta_{\varepsilon}^{(i)}(t)=\int_{0}^{t} \Theta_{i}\left(\frac{\omega_{1} s}{\varepsilon^{k}}, \frac{\omega_{2} s}{\varepsilon^{k}}\right) d M_{\varepsilon}(s), i=\overline{1,4}, \\
d M_{\varepsilon}(t)=\sum_{i=1}^{m} \varepsilon^{k_{i}-k / 2} \hat{f}_{i}\left(\frac{\mu_{i} t}{\varepsilon^{k}}, A_{\varepsilon}(t), \frac{\omega_{1} t}{\varepsilon^{k}}, \frac{\omega_{2} t}{\varepsilon^{k}}\right) d w_{i}^{\varepsilon}(t)+\int_{\mathbb{R}} \hat{f}_{m+1}\left(\frac{\mu_{m+1} t}{\varepsilon^{k}}, A_{\varepsilon}(t), \frac{\omega_{1} t}{\varepsilon^{k}}, \frac{\omega_{2} t}{\varepsilon^{k}}, z\right) \tilde{\nu}_{1}^{\varepsilon}(d t, d z) \\
+\varepsilon^{k_{m+2}} \int_{\mathbb{R}} \hat{f}_{m+2}\left(\frac{\mu_{m+2} t}{\varepsilon^{k}}, A_{\varepsilon}(t), \frac{\omega_{1} t}{\varepsilon^{k}}, \frac{\omega_{2} t}{\varepsilon^{k}}, z\right) \tilde{\nu}_{2}^{\varepsilon}(d t, d z),
\end{gathered}
$$

and derive, that for stochastic processes $A_{\varepsilon}(t), \zeta_{\varepsilon}(t)$ conditions of weak compactness [20] holds true. So for any sequence $\varepsilon_{n} \rightarrow 0, n=1,2, \ldots$ there exists a subsequence $\varepsilon_{m}=\varepsilon_{n(m)} \rightarrow 0, m=1,2, \ldots$, probability space, stochastic processes $\bar{A}_{\varepsilon_{m}}(t)=\left(\bar{A}_{11}^{\varepsilon_{m}}(t), \bar{A}_{12}^{\varepsilon_{m}}(t), \bar{A}_{21}^{\varepsilon_{m}}(t), \bar{A}_{22}^{\varepsilon_{m}}(t)\right), \bar{\zeta}_{\varepsilon_{m}}(t), \bar{A}(t)=\left(\bar{A}_{11}(t), \bar{A}_{12}(t), \bar{A}_{21}(t), \bar{A}_{22}(t)\right)$, $\bar{\zeta}(t)$ defined on this space, such that $\bar{A}_{\varepsilon_{m}}(t) \rightarrow \bar{A}(t), \bar{\zeta}_{\varepsilon_{m}}(t) \rightarrow \bar{\zeta}(t)$ in probability, as $\varepsilon_{m} \rightarrow 0$, and finitedimensional distributions of $\bar{A}_{\varepsilon_{m}}(t), \bar{\zeta}_{\varepsilon_{m}}(t)$ are coincide with finite-dimensional distributions of $A_{\varepsilon_{m}}(t), \zeta_{\varepsilon_{m}}(t)$. Since we are interested in limit behaviour of distributions, we can consider processes $A_{\varepsilon_{m}}(t)$ and $\zeta_{\varepsilon_{m}}(t)$ instead of $\bar{A}_{\varepsilon_{m}}(t), \bar{\zeta}_{\varepsilon_{m}}(t)$. From (24) we obtain equation

$$
A_{\varepsilon_{m}}(t)=A(0)+\int_{0}^{t} \alpha_{\varepsilon_{m}}\left(s, A_{\varepsilon_{m}}(s)\right) d s+\zeta_{\varepsilon_{m}}(t), \quad A(0)=\left(A_{i}(0), i=\overline{1,4}\right),
$$

where $\alpha_{\varepsilon}(t, A)=\left(\alpha_{\varepsilon}^{(i)}(t, A), i=\overline{1,4}\right)$,

$$
\begin{aligned}
& \alpha_{\varepsilon}^{(i)}(t, A)= \\
& =\Theta_{i}\left(\frac{\omega_{1} t}{\varepsilon^{k}}, \frac{\omega_{2} t}{\varepsilon^{k}}\right)\left[\varepsilon^{k_{0}-k} \hat{f}_{0}\left(\frac{\mu_{0} t}{\varepsilon^{k}}, A, \frac{\omega_{1} t}{\varepsilon^{k}}, \frac{\omega_{2} t}{\varepsilon^{k}}\right)+\varepsilon^{k_{m+2}-k} \int_{\mathbb{R}} \hat{f}_{m+2}\left(\frac{\mu_{m+2} t}{\varepsilon^{k}}, A, \frac{\omega_{1} t}{\varepsilon^{k}}, \frac{\omega_{2} t}{\varepsilon^{k}}, z\right) \Pi_{2}(d z)\right],
\end{aligned}
$$

$i=\overline{1,4}$.

Also the process $\zeta_{\varepsilon}(t)$ is the vector-valued square integrable martingale with matrix characteristic

$$
\begin{aligned}
& \left\langle\zeta_{\varepsilon}^{(l)}, \zeta_{\varepsilon}^{(n)}\right\rangle(t)=\sum_{j=1}^{m} \int_{0}^{t} \sigma_{\varepsilon}^{(l, j)}\left(s, A_{\varepsilon}(s)\right) \sigma_{\varepsilon}^{(n, j)}\left(s, A_{\varepsilon}(s)\right) d s \\
& +\frac{1}{\varepsilon^{k}} \int_{0}^{t} \int_{\mathbb{R}} \gamma_{\varepsilon}^{(l)}\left(s, A_{\varepsilon}(s), z\right) \gamma_{\varepsilon}^{(n)}\left(s, A_{\varepsilon}(s), z\right) \Pi_{1}(d z) d s \\
& +\frac{1}{\varepsilon^{k}} \int_{0}^{t} \int_{\mathbb{R}} \delta_{\varepsilon}^{(l)}\left(s, A_{\varepsilon}(s), z\right) \delta_{\varepsilon}^{(n)}\left(s, A_{\varepsilon}(s), z\right) \Pi_{2}(d z) d s, l, n=\overline{1,4},
\end{aligned}
$$

where

$$
\begin{aligned}
& \sigma_{\varepsilon}^{(l, j)}(s, A)=\varepsilon^{k_{j}-k / 2} \Theta_{l}\left(\frac{\omega_{1} s}{\varepsilon^{k}}, \frac{\omega_{2} s}{\varepsilon^{k}}\right) \hat{f}_{j}\left(\frac{\mu_{j} s}{\varepsilon^{k}}, A, \frac{\omega_{1} s}{\varepsilon^{k}}, \frac{\omega_{2} s}{\varepsilon^{k}}\right), \\
& \gamma_{\varepsilon}^{(l)}(s, A, z)=\varepsilon^{k_{m+1}} \Theta_{l}\left(\frac{\omega_{1} s}{\varepsilon^{k}}, \frac{\omega_{2} s}{\varepsilon^{k}}\right) \hat{f}_{m+1}\left(\frac{\mu_{m+1} s}{\varepsilon^{k}}, A, \frac{\omega_{1} s}{\varepsilon^{k}}, \frac{\omega_{2} s}{\varepsilon^{k}}, z\right), \\
& \delta_{\varepsilon}^{(l)}(s, A, z)=\varepsilon^{k_{m+2}} \Theta_{l}\left(\frac{\omega_{1} s}{\varepsilon^{k}}, \frac{\omega_{2} s}{\varepsilon^{k}}\right) \hat{f}_{m+2}\left(\frac{\mu_{m+2} s}{\varepsilon^{k}}, A, \frac{\omega_{1} s}{\varepsilon^{k}}, \frac{\omega_{2} s}{\varepsilon^{k}}, z\right), \quad l=\overline{1,4} .
\end{aligned}
$$


For the processes $A_{\varepsilon_{m}}(t)$ and $\zeta_{\varepsilon_{m}}(t)$ the estimates $(15)$ - (17) hold true, so processes $\bar{A}(t)$ and $\bar{\zeta}(t)$ satisfy the Kolmogorov's continuity condition [21].

Let us consider the case $k_{0}=2 k_{i}=k_{m+2}, i=\overline{1, m+1}$ and $\mu_{j}=\frac{p_{j}^{(1)}}{q_{j}^{(1)}} \omega_{1}=\frac{p_{j}^{(2)}}{q_{j}^{(2)}} \omega_{2}$ for all $j=\overline{0, m+2}$, where $p_{j}^{(i)}$ and $q_{j}^{(i)}$ are some relatively prime integers, $i=1,2, j=\overline{0, m+2}$. Under these conditions from Fourier series expansion of integrand functions on variables $\mu_{j} t / \varepsilon^{k}, j=\overline{0, m+2}, \omega_{1} t / \varepsilon^{k}$ and $\omega_{2} t / \varepsilon^{k}$ in corresponding terms, we obtain for $l, n=\overline{1,4}$

$$
\begin{aligned}
& \lim _{\varepsilon \rightarrow 0} \frac{1}{t} \int_{0}^{t} \alpha_{\varepsilon}^{(l)}(s, A) d s=\bar{\alpha}_{l}(A), \\
& \lim _{\varepsilon \rightarrow 0} \frac{1}{t} \int_{0}^{t}\left[\sum_{j=1}^{m} \sigma_{\varepsilon}^{(l, j)}(s, A) \sigma_{\varepsilon}^{(n, j)}(s, A)+\frac{1}{\varepsilon^{k}} \int_{R} \gamma_{\varepsilon}^{(l)}(s, A, z) \gamma_{\varepsilon}^{(n)}(s, A, z) \Pi_{1}(d z)\right. \\
& \left.+\frac{1}{\varepsilon^{k}} \int_{R} \delta_{\varepsilon}^{(l)}(s, A, z) \delta_{\varepsilon}^{(n)}(s, A, z) \Pi_{2}(d z)\right] d s=\bar{B}_{l n}(A),
\end{aligned}
$$

where functions $\bar{\alpha}_{l}(A), l=\overline{1,4}$ and $\bar{B}(A)=\left\{\bar{B}_{l n}(A), l, n=\overline{1,4}\right\}$ are defined in the conditions of theorem.

Since $A_{\varepsilon_{m}}(t) \rightarrow \bar{A}(t), \zeta_{\varepsilon_{m}}(t) \rightarrow \bar{\zeta}(t)$ in probability, as $\varepsilon_{m} \rightarrow 0$, processes $\bar{A}(t), \bar{\zeta}(t)$ are continuous, functions $f_{j}, j=\overline{0, m+2}$ bounded and satisfy Lipschitz condition on $y_{i}, i=\overline{1,4}$, function $\Theta\left(\phi_{1}, \phi_{2}\right)$ is bounded, then from Lemma 1, Remark 1 and relationships (25), (26) it follows

$$
\bar{A}(t)=A(0)+\int_{0}^{t} \bar{\alpha}(\bar{A}(s)) d s+\bar{\zeta}(t),
$$

almost surely, where $\bar{\zeta}(t)=\left(\bar{\zeta}^{(i)}(t), i=\overline{1,4}\right)$ is continuous vector-valued martingale with matrix characteristic

$$
\left\langle\bar{\zeta}^{(i)}, \bar{\zeta}^{(j)}\right\rangle(t)=\int_{0}^{t} \bar{B}_{i j}(\bar{A}(s)) d s, \quad i, j=\overline{1,4} .
$$

Hence [22] there exists Wiener process $\bar{w}(t)=\left(\bar{w}_{i}(t), i=\overline{1,4}\right)$, such that

$$
\bar{\zeta}(t)=\int_{0}^{t} \bar{\sigma}(\bar{A}(s)) d \bar{w}(s), \quad \bar{\sigma}(A)=\{\bar{B}(A)\}^{1 / 2} .
$$

Relationships (27), (28) mean that process $\bar{A}(t)$ satisfies equation (23). Under conditions of theorem the equation (23) has unique solution. Therefore process $\bar{A}(t)$ does not depend on choosing of sub-sequence $\varepsilon_{m} \rightarrow 0$, and finite-dimensional distributions of process $A_{\varepsilon_{m}}(t)$ converge to finite-dimensional distributions of process $\bar{A}(t)$. Since processes $A_{\varepsilon_{m}}(t)$ and $\bar{A}(t)$ are Markov processes then using the conditions for weak convergence of Markov processes [21] we finish the proof of statement 1) of the theorem.

Statements 2) -4) of the theorem follow from (26) by simple analysis. Statement 5) of the theorem is proved by the same argument as in the proof of statement 2) of the Theorem 3.

\section{REFERENCES}

1. Ping Chen, Sardar M.N. Islam, Optimal Control Models in Finance, Springer, 2005.

2. C. Ye, J.P. Huang, Non-classical oscillator model for persistent fluctuations in stock markets, Physica A, 387, pp. 1255 - 1263, 2008. 
3. N.M. Krylov, and N.N. Bogolyubov, Introduction to Non-Linear Mechanics, Princeton University Press, 1949.

4. N.N. Bogoliubov, and Yu.A. Mitropolsky, Asymptotic Methods in Theory of Non-Linear Oscillations, Gordon and Breach Science Publishers, 1985.

5. Yu.A. Mitropolskij, and V.G. Kolomiets, On the action of random forces on the non-linear oscillation systems, Math. Physics and Non-Linear Mechanics, v.5(39), pp.23 -34, 1986. (In Russian)

6. O.V. Borysenko, Small random desturbances in second order oscillating systes, Ukrainian Mathematical Journal, v.44, ?, pp.11 17, 1992.(In Russian)

7. O.V. Borysenko, Behaviour of non-autonomous oscillating system under the action of small random disturbances in resonance case, Ukrainian Mathematical Journal, v.44, ?2, pp. 1645 - 1651, 1992. (In Russian)

8. O.D. Borysenko, and O.V. Borysenko, Asymptotic behaviour of stochastic dynamic system, Theory of Optimal Dicisions, Kiev, Inst.Kibernetiki NANU, pp. 38 - 44, 1999. (In Russian)

9. O.D. Borysenko, O.V. Borysenko, and I.G. Malyshev, Limit behaviour of dynamic system under the action of small random disturbances in non-resonance case, Theory of Optimal Dicisions, Kyiv, Inst.Cibernetics NANU, ?, pp. 70 - 78, 2002. (In Russian)

10. O.D. Borysenko, O.V. Borysenko, and I.G. Malyshev, Asymptotic behavior of third order random oscillation system in the nonresonance case, Theory of Stochastic Processes, 9(25), , ?-4, pp. 1 - 11, 2003.

11. O.D. Borysenko, O.V. Borysenko, and D.O. Borysenko, Averaging method for autonomous third order random oscillating system, Bulletin of Taras Shevchenko National University of Kyiv. Series: Physics \& Mathematics, ?, pp. 7 - 11, 2013.

12. O.D. Borysenko, and O.V. Borysenko, Averaging method for non-autonomous third order random oscillating system in nonresonance case, Bulletin of Taras Shevchenko National University of Kyiv. Series: Physics \& Mathematics, ?, pp. 7 - 11, 2014.

13. O.D. Borysenko, and O.V. Borysenko, Averaging method for autonomous fourth order oscillating system under the action of random disturbances, Bulletin of Taras Shevchenko National University of Kyiv. Series: Physics \& Mathematics, ?, pp. 11 - 15, 2010.

14. O.D. Borysenko, and O.V. Borysenko, Limit behaviour of autnomous fourth order oscillating system under the action of random disturbances in the case of complex roots to characteristic equation, Bulletin of Taras Shevchenko National University of Kyiv Series: Physics \& Mathematics, ?, pp. 7 - 11, 2011.

15. O.D. Borysenko, and O.V. Borysenko, Limit behavior of autonomous fours order oscillating system under the action of random perturbations in the case of two pairs of imaginary adjoined roots of characteristic equation, Bulletin of Taras Shevchenko National University of Kyiv. Series: Physics \& Mathematics, ?, pp. 10 - 14, 2012.

16. A. M. Samoilenko, O. M. Stanzhytskij, and N. I. Makhmudov, The averaging method and two-sided bounded solutions of Ito stochastic systems, Differential Equations, 43(1), pp. 56 - 68, 2007.

17. O.D. Borysenko, and O.V. Borysenko, Non-autonomous fourth order random oscillating systems under the action of external periodical disturbances, Bulletin of Taras Shevchenko National University of Kyiv. Series: Physics \& Mathematics, ?, pp. 9 - 14, 2016.

18. O.D. Borysenko, and O.V. Borysenko, Investigation of non-autonomous fourth order random oscillating systems in the case of complex roots of characteristic equation, Bulletin of Taras Shevchenko National University of Kyiv. Series: Physics \& Mathematics, ?, pp. $9-12,2017$.

19. O.D. Borysenko, and I.G. Malyshev The limit behaviour of integral functional of the solution of stochastic differential equation depending on small parameter, Theory of Stochastic Processes, Vol. 7(23), no. 1-2, pp. 30-36, 2001.

20. A.V. Skorokhod, Studies in the Theory of Random Processes, Addison-Wesley, 1965.

21. I.I. Gikhman, and A.V. Skorokhod, Theory of Random Processes, v.1, M.: Nauka, 1971. (In Russian)

22. N. Ikeda, and S. Watanabe, Stochastic Differential Equations and Diffusion Processes North Holland, Amsterdam and Kadansha, Tokyo, 1981. 